\title{
Myeloperoxidase, paraoxonase-1, and HDL form a functional ternary complex
}

Ying Huang, ${ }^{1}$ Zhiping Wu, ${ }^{1}$ Meliana Riwanto,, ${ }^{2,3}$ Shengqiang Gao, ${ }^{1}$ Bruce S. Levison, ${ }^{1}$ Xiaodong Gu, Xiaoming Fu, ${ }^{1}$ Matthew A. Wagner, ${ }^{1}$ Christian Besler, ${ }^{2,3}$ Gary Gerstenecker, ${ }^{4}$ Renliang Zhang, ${ }^{1}$ Xin-Min Li, ${ }^{1}$ Anthony J. DiDonato, ${ }^{1,5}$ Valentin Gogonea, ${ }^{1,4}$ W.H. Wilson Tang, ${ }^{1,6}$ Jonathan D. Smith, ${ }^{1,6}$ Edward F. Plow, ${ }^{6,7}$ Paul L. Fox, ${ }^{1}$ Diana M. Shih, ${ }^{8}$ Aldons J. Lusis, ${ }^{8}$ Edward A. Fisher, ${ }^{9}$ Joseph A. DiDonato, ${ }^{1}$ Ulf Landmesser, ${ }^{2,3}$ and Stanley L. Hazen ${ }^{1,6}$

1Department of Cellular and Molecular Medicine, Center for Cardiovascular Diagnostics and Prevention, Cleveland Clinic, Cleveland, Ohio, USA. ${ }^{2}$ Cardiology, Cardiovascular Center, University Hospital Zurich, and ${ }^{3}$ Cardiovascular Research, Institute of Physiology, and Center for Integrative Human Physiology, University of Zurich, Zurich, Switzerland. ${ }^{4}$ Department of Chemistry, Cleveland State University, Cleveland, Ohio, USA. ${ }^{5}$ Department of Psychology, John Carroll University, University Heights, Ohio, USA. ${ }^{6}$ Department of Cardiovascular Medicine and ${ }^{7}$ Department of Molecular Cardiology, Cleveland Clinic, Cleveland, Ohio, USA. ${ }^{8}$ Department of Medicine/Division of Cardiology, BH-307 Center for the Health Sciences, UCLA, Los Angeles, California, USA. ${ }^{9}$ Department of Medicine, New York University, New York, New York, USA.

\begin{abstract}
Myeloperoxidase (MPO) and paraoxonase 1 (PON1) are high-density lipoprotein-associated (HDL-associated) proteins mechanistically linked to inflammation, oxidant stress, and atherosclerosis. MPO is a source of ROS during inflammation and can oxidize apolipoprotein A1 (APOA1) of HDL, impairing its atheroprotective functions. In contrast, PON1 fosters systemic antioxidant effects and promotes some of the atheroprotective properties attributed to HDL. Here, we demonstrate that MPO, PON1, and HDL bind to one another, forming a ternary complex, wherein PON1 partially inhibits MPO activity, while MPO inactivates PON1. MPO oxidizes PON1 on tyrosine 71 ( $\left.\mathrm{Tyr}_{71}\right)$, a modified residue found in human atheroma that is critical for HDL binding and PON1 function. Acute inflammation model studies with transgenic and knockout mice for either PON1 or MPO confirmed that MPO and PON1 reciprocally modulate each other's function in vivo. Further structure and function studies identified critical contact sites between APOA1 within HDL, PON1, and MPO, and proteomics studies of HDL recovered from acute coronary syndrome (ACS) subjects revealed enhanced chlorotyrosine content, site-specific PON1 methionine oxidation, and reduced PON1 activity. HDL thus serves as a scaffold upon which MPO and PON1 interact during inflammation, whereupon PON1 binding partially inhibits MPO activity, and MPO promotes site-specific oxidative modification and impairment of PON1 and APOA1 function.
\end{abstract}

\section{Introduction}

Cholesterol efflux activity and reverse cholesterol transport are regarded as the classic functions of high-density lipoprotein (HDL). Yet increasing evidence shows that additional cardioprotective antioxidant and antiinflammatory functions of HDL exist $(1,2)$. Isolated HDL can reduce LDL-associated cholesteryl ester hydroperoxides, fatty acid hydroperoxides, and certain oxidized phosphatidylcholine molecular species (3). Moreover, HDL facilitates numerous antiinflammatory activities (4-7). Interestingly, reconstituted particles containing apolipoprotein A1 (APOA1), the major structural protein within $\mathrm{HDL}$, can recapitulate some but not all of these activities (6-8). Thus, the antiinflammatory

Conflict of interest: Drs. Hazen and Smith report being listed as coinventors on pending and issued patents held by the Cleveland Clinic relating to cardiovascular diagnostics and therapeutics. Dr. Hazen reports having been paid as a consultant for the following companies: Abbott, Cleveland Heart Lab, Esperion, Lilly, LipoScience Inc., Merck \& Co., Inc., and Pfizer Inc. Dr. Hazen reports receiving research funds from Abbott, Cleveland Heart Lab, LipoScience Inc., Proctor \& Gamble, and Roche. Dr. Hazen reports having the right to receive royalty payments for inventions or discoveries related to cardiovascular diagnostics or therapeutics from the following companies: Abbott, Cleveland Heart Lab, Esperion, Frantz Biomarkers, LLC, LipoScience Inc., and Siemens. Dr. Smith reports having the right to receive royalty payments for inventions or discoveries related to cardiovascular diagnostics or therapeutics from Cleveland Heart Lab and Esperion. Dr. Levison reports having the right to receive royalty payments for inventions or discoveries related to cardiovascular diagnostics from LipoScience Inc. Dr. Tang reports having previously received research grant support from Abbott Laboratories.

Citation for this article: J Clin Invest. 2013;123(9):3815-3828. doi:10.1172/JCI67478. and antioxidant activities of HDL are thought to be mediated in part by both APOA1 within the core lipoprotein particle and an assortment of HDL-associated proteins.

Early proteomics studies of HDL provided the first glimpse into the complexity of the HDL-associated proteome, revealing HDL as a highly heterogeneous array of distinct particles that are equipped with a broad assortment of HDL-associated proteins, including not only previously recognized exchangeable apolipoproteins, but also numerous additional proteins involved in hemostasis, thrombosis, the immune and complement systems, growth factors, receptors, and hormone-associated proteins $(9,10)$. Subsequent studies both replicated these findings and extended them by suggesting that the composition of HDL-associated proteins may differ between subjects having clinically significant cardiovascular disease compared with healthy controls (11-13). This raises the possibility that alterations in HDL function may occur through changes in HDL-associated proteins. Indeed, the contemporary view holds that distinct HDL particle subpopulations exist, which are composed of unique clusters of specific HDL-associated proteins that foster specific biological functions (12). For example, HDL particles enriched in paraoxonase 1 (PON1), an atheroprotective protein (14), have been linked to the antioxidative, antiinflammatory, and lipid cargo-carrying functions of $\operatorname{HDL}(7,12,15)$. An esterase/lipolactonase with unclear substrate(s) in vivo, PON1 has been shown to help HDL prevent the accumulation of lipid peroxides in oxidized LDL, inactivate bioactive oxidized phospholipids, 
stimulate HDL-mediated eNOS-dependent NO production, and enhance cholesterol efflux from cholesterol-laden macrophages $(7,16-18)$. The participation of PON1 in HDL-related beneficial functions extends to in vivo studies as well, since PON1 KO mice show enhanced susceptibility for developing atherosclerosis (19) and, conversely, Pon1 transgenic (Tg) mice demonstrate both protection from the development of atherosclerosis and reduced systemic measures of oxidation (20). Moreover, recent human studies show a striking correlation between multiple systemic measures of oxidant stress and serum PON1 activity measures (15), and reduced systemic PON1 activity heralds increased prospective risks for major adverse cardiac events in subjects $(15,21$, 22). PON1 has thus emerged as an important mediator of many antiinflammatory, antioxidant, and atheroprotective activities of HDL. A role for an HDL-associated protein in modulating PON1 activity levels within a distinct HDL particle subpopulation has not yet been reported.

Myeloperoxidase (MPO) is a leukocyte-derived heme protein that, like PON1, both binds to HDL and is mechanistically linked to oxidant stress and atherosclerosis (23-27). While typically found at very low levels within the plasma of healthy individuals, substantially elevated levels may be observed in the plasma or serum of those with acute coronary syndromes (ACS) and at risk for major adverse cardiac events (28). An enzymatic source of diffusible oxidant species, MPO promotes protein and lipid oxidation at sites of inflammation, including within the atherosclerotic plaque-laden artery where the enzyme is enriched and catalytically active $(24,29-32)$. Studies using neutrophils (PMN) isolated from subjects with MPO deficiency $\left(\mathrm{PMN}_{\mathrm{MPO}}\right)$ reveal that MPO is also a major enzymatic catalyst for the initiation of lipid peroxidation within serum or plasma (33), and studies employing MPO KO mice confirm that the leukocyte enzyme plays a dominant role in lipid peroxidation and eicosanoid formation at sites of acute inflammation (34). When released from activated leukocytes, MPO within both the circulation and within human atherosclerotic plaque binds to HDL, targeting the particle for oxidative modification and functional inactivation $(6,23,35)$. Site-specific oxidation of APOA1 of HDL by MPO has been linked to impairment in cholesterol efflux and LCAT-activating activities of the lipoprotein, inhibition in antiinflammatory and antiapoptotic properties of HDL, and acquisition of proinflammatory gain-offunction activities $(6,23)$.

The processes that regulate MPO activity in vivo are unclear. Our current understanding is that the availability of MPO is regulated predominantly by the extent of leukocyte activation and granule content secretion, and catalytic activity of released MPO is governed in large part by hydrogen peroxide substrate availability. Whether MPO activity is further modulated by interaction with additional proteins, such as after binding to the HDL particle or a specific HDL-associated protein, is not known. Given the numerous mechanistic and opposing links between MPO and PON1 with systemic measures of oxidant stress, inflammation, and atherosclerotic risk, we hypothesized that these two HDL-associated proteins may functionally interact and inhibit each other's catalytic activity either on HDL or outside of their association with HDL.

\section{Results}

$M P O$ and PON1 bind to one another and reciprocally inbibit each other's activity. To test the hypothesis that HDL, PON1, and MPO modulate each other's activity, we initially examined whether immunoprecipitation studies were consistent with a potential complex being formed between HDL, MPO, and PON1 in plasma. As expected, antibodies against APOA1 pulled down both MPO and PON1 from plasma (Supplemental Figure 1; supplemental material available online with this article; doi:10.1172/ JCI67478DS1). Importantly, cross immunoprecipitation studies in plasma with antibodies against either MPO or PON1 were also consistent with ternary complex formation involving HDL (APOA1), MPO, and PON1, since immunoprecipitation with antibodies against either MPO or PON1 similarly pulled down the other 2 components (Supplemental Figure 1). To more fully assess the strength of the interactions between each component of a presumptive HDL-MPO-PON1 complex, plasmon resonance spectroscopy was performed to evaluate binding interactions between each of the components. Remarkably, HDL, MPO, and PON1 each bound to one another with high affinity, showing $K_{\mathrm{D}}$ values in the low nanomolar-to-picomolar range, depending on the interaction examined (Supplemental Figure 2).

PON1 in plasma is present on a subset of HDL particles (estimated at approximately 1 of $8-10 \mathrm{HDL}$ particles) (36). And MPO is significantly less abundant than either PON1 or HDL in plasma. Western blot analyses with MPO antibodies following immunoprecipitation with either PON1 or APOA1 reveal that the vast majority of MPO in plasma is bound to HDL and PON1, as nominal detectable MPO remains in the supernatant following immunoprecipitation with antibodies against APOA1 and PON1 (not shown). The above binding studies suggest that MPO, when present within plasma or serum or within other bodily tissues or fluids, may interact with APOA1 and PON1 in a subset of HDL particles. The ability of MPO to oxidize APOA1 and alter HDL function is well known $(6,23)$. Whether interactions between MPO and PON1 might alter the function of each of these HDL-associated proteins, however, is unclear. We therefore next examined whether MPO activity (using varying levels of $\mathrm{H}_{2} \mathrm{O}_{2}$ ) impacted PON1 activity within a complex matrix-like serum. Physiological levels of HDL (1 mg protein/ml) and PON1 $(100 \mu \mathrm{g} / \mathrm{ml})$ were added to lipoprotein-deficient serum from a healthy volunteer in which the MPO levels measured were nominal ( $<50$ picomolar). In the absence of additional MPO, increasing amounts of the MPO substrate $\mathrm{H}_{2} \mathrm{O}_{2}$ had minimal effect on PON1 activity. In contrast, upon the addition of pathophysiological levels of MPO (e.g., $5 \mathrm{nM}$ ), a dose-dependent inhibition of PON1 catalytic activity was observed with increasing levels of $\mathrm{H}_{2} \mathrm{O}_{2}$ (Figure 1A). The addition of a large molar excess of methionine, a scavenger of hypochlorous acid $(\mathrm{HOCl})$ - the major oxidant formed from MPO in the presence of plasma levels of chloride - blocked the inhibitory effect of $\mathrm{MPO} / \mathrm{H}_{2} \mathrm{O}_{2}$ on PON1, in agreement with $\mathrm{HOCl}$ as the reactive oxidant responsible for the loss of PON1 catalytic activity. Consistent with this, in a parallel study, $\mathrm{HOCl}$ was incrementally added to lipoprotein-deficient serum supplemented with HDL and PON1 in the absence of MPO. Again, a dose-dependent inhibition of PON1 catalytic activity was observed (Figure 1A). Importantly, comparisons between the $\mathrm{MPO} / \mathrm{H} 2 \mathrm{O} 2 / \mathrm{Cl}^{-}$system (an enzymatic source of $\mathrm{HOCl}$ ) and $\mathrm{HOCl}$ at molar-equivalent doses demonstrated MPO to be a more potent inhibitor of PON1 activity at every level of oxidant examined, consistent with MPO binding to the HDL-PON1 complex within serum and the reactive $\mathrm{HOCl}$ formed by MPO having a shorter diffusion distance (and hence, less scavenging) before hitting the PON1 target (Figure 1A). 
A

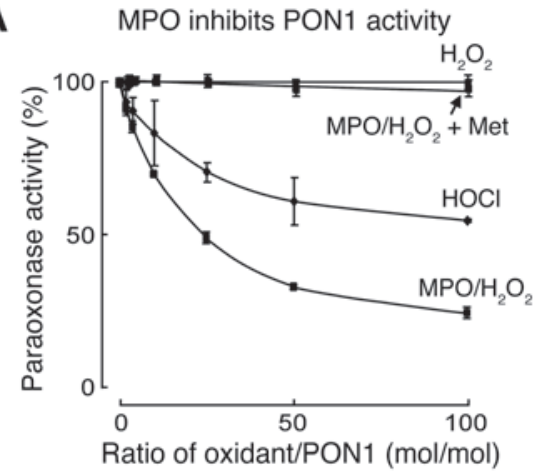

B

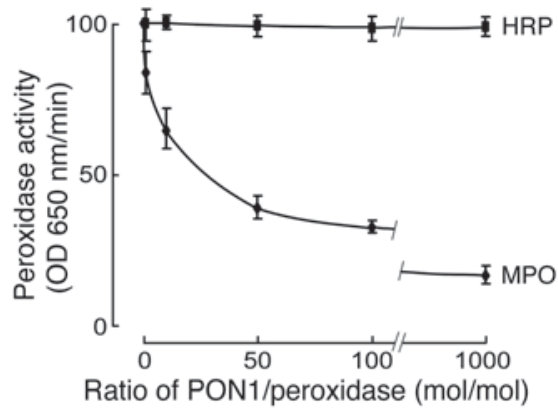

C

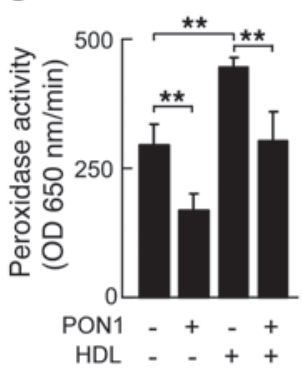

D

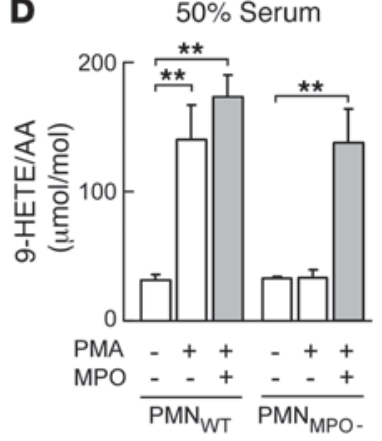

E

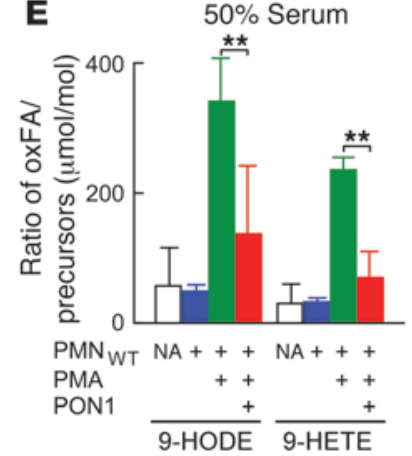

$\mathbf{F}$

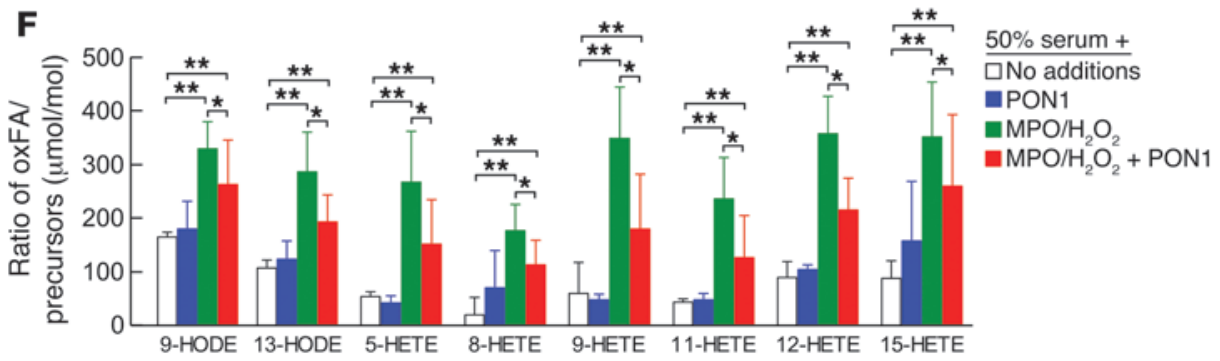

Figure 1

MPO and PON1 inhibit each other's activity in vitro. (A) PON1 $(100 \mu \mathrm{g} / \mathrm{ml})$ was incubated with either $\mathrm{HOCl}$ or $\mathrm{H}_{2} \mathrm{O}_{2}$ and MPO $(50 \mathrm{nM})$ in $50 \mathrm{mM} \mathrm{Na}\left[\mathrm{PO}_{4}\right]$ buffer (pH 7.0) supplemented with isolated human $\mathrm{HDL}(1 \mathrm{mg} / \mathrm{ml})$ and $100 \mathrm{mM} \mathrm{NaCl}$ at $37^{\circ} \mathrm{C}$ for 60 minutes. This was followed by quantification of paraoxonase activity relative to no oxidant exposures. Also shown are the effects of varying levels of $\mathrm{H}_{2} \mathrm{O}_{2}$ alone (i.e., no MPO added) or with the addition of the $\mathrm{HOCl}$ scavenger methionine (Met) (10-fold molar excess relative to oxidant) to the MPO/ $\mathrm{H}_{2} \mathrm{O}_{2}$ system. (B) Effect of $\mathrm{PON} 1$ on peroxidase activity by $\mathrm{TMB}$ assay of either MPO or HRP. (C) Effect by TMB assay of PON1 (650 nM), HDL (650 nM), or both on MPO $\left(65 \mathrm{nM}\right.$ ) peroxidase activity. (D and E) Human neutrophils isolated from healthy donors (PMN $\mathrm{WT}_{\mathrm{WT}}$ or MPO-deficient subjects ( $\mathrm{PMN}_{\mathrm{MPO}}$ ) were incubated at $37^{\circ} \mathrm{C}$ in $50 \%$ serum for 1 hour in the absence or presence of phorbol 12-myristrate 13-acetate (PMA) and MPO or PON1, as indicated. Endogenous serum arachidonic acid (AA), linoleic acid (LA), 9-HODE, and 9-HETE were then quantified by stable isotope dilution LC/MS/MS as described in Methods. (F) PON1, MPO/ $\mathrm{H}_{2} \mathrm{O}_{2}$, neither, or both were added to $50 \%$ serum, incubated at $37^{\circ} \mathrm{C}$ for 1 hour, and then $A A, L A$, or the indicated oxidation products were quantified as described in Methods. Data shown represent the mean \pm SD of triplicate determinations. ${ }^{*} P<0.05$; ${ }^{* *} P<0.001$. NA, no additions; oxFA, oxidized fatty acids.

We next sought to test whether PON1 could influence MPO catalytic activity. The addition of PON1 to MPO inhibited peroxidase activity by approximately $50 \%$ (Figure $1 \mathrm{~B}$ ). The effect of PON1 on MPO peroxidase activity was specific, since the replacement of MPO with an alternative heme peroxidase, HRP (but the same levels of $\mathrm{H}_{2} \mathrm{O}_{2}$ and chromophore substrate), showed no effect of PON1 on peroxidase activity (Figure 1B). Interest- ingly, the addition of HDL to MPO resulted in a reproducible, modest increase in MPO peroxidase activity; however, the addition of PON1 in the presence of HDL still resulted in a reduction in MPO peroxidase activity by approximately $50 \%$ (Figure $1 \mathrm{C}$ ). To further explore the physiological relevance of MPO-PON1 interaction, additional studies were performed. We previously used isolated human neutrophils from normal and MPOdeficient subjects to show that neutrophil activation in plasma or serum is a physiological mechanism for the initiation of lipid peroxidation, such as at sites of acute inflammation, through a mechanism requiring MPO (33). We therefore examined the impact of PON1 on human neutrophil-dependent lipid peroxidation in serum as monitored by stable isotope dilution liquid chromatography tandem mass spectrometry (LC/ MS/MS) quantification of oxidation products of arachidonic (e.g., hydroxyeicosatetraenoic acid [HETEs]) and linoleic acids (e.g., hydroxyoctadecadienoic acid [HODEs]). As expected, the addition of neutrophils from normal volunteers $\left(\mathrm{PMN}_{\mathrm{WT}}\right)$ to serum failed to promote lipid peroxidation under basal conditions, but did so following neutrophil activation by the addition of phorbol ester agonist (Figure 1D). As an additional control to show that the lipid peroxidation monitored was attributable to MPO release from the PMN, parallel studies using neutrophils isolated from an MPO-deficient subject (PMN $\left.\mathrm{MPO}_{\mathrm{MPO}}\right)$ were performed, demonstrating no formation of 9-HETE (a free radical-specific oxidation product of arachidonic acid) following the addition of phorbol ester agonist, but marked 9-HETE formation upon the addition of catalytic levels of MPO to activated PMN $\mathrm{NPO}_{\text {- }}$ (Figure 1D). The addition of PON1 to serum in the presence of quiescent PMN $\mathrm{WT}_{\mathrm{W}}$ resulted in no change in 9-HODE or 9-HETE levels, but markedly attenuated the incremental increases in lipid peroxidation products formed upon PMN $\mathrm{WT}_{\mathrm{WT}}$ stimulation (Figure 1E). In a parallel set of studies, the direct impact of PON1 on the extent of MPO-catalyzed formation of multiple distinct molecular species of HETEs and HODEs in serum was determined by stable isotope dilution LC/MS/MS. Again, the addition of PON1 markedly attenuated MPO-dependent initiation of lipid peroxidation and formation of 
A

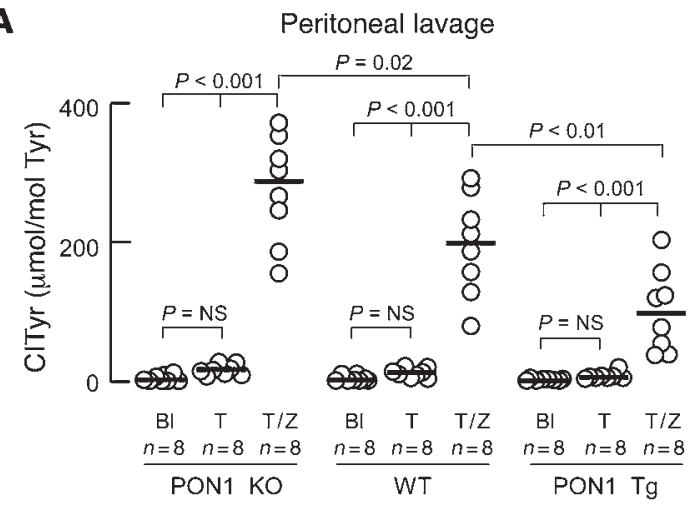

B

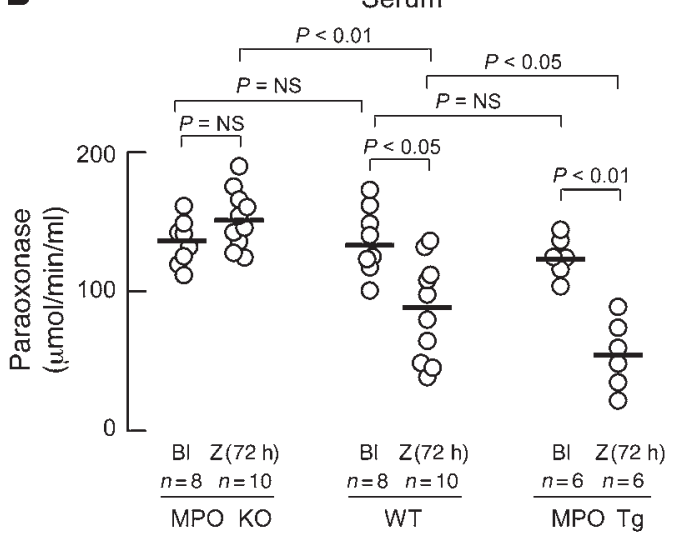

the various HETEs and HODEs from endogenous lipids in serum (Figure 1F). Collectively, these results indicate that within a physiological matrix like plasma or serum, MPO and PON1 can interact with one another and modulate each other's activity.

$M P O$ and PON1 influence each other's activity in vivo. To further extend our observations of the reciprocal inhibitory effects of $\mathrm{MPO}$ and PON1 on each other's activity from an in vitro (ex vivo) to an in vivo setting, further studies were performed using mice genetically engineered to lack $(\mathrm{KO})$ or overexpress $(\mathrm{Tg})$ either MPO or PON1. We chose a simple and well-characterized peritonitis model of acute inflammation for these studies, since we have previously shown that MPO serves as a major enzymatic catalyst for both protein and lipid oxidation within the model $(31,34)$. To examine the impact of PON1 on MPO activity, we quantified protein-bound 3-chlorotyrosine (ClTyr), a specific molecular fingerprint for MPO-catalyzed protein oxidation (29, 31 ), in peritoneal proteins recovered from PON1 KO, WT, and PON1 Tg mice following the recruitment of leukocytes to the peritoneal cavity with thioglycolate and subsequent activation by i.p. injection of yeast cell wall protein carbohydrate components (zymosan). Compared with WT, the PON1 KO mice demonstrated a significant $(P=0.02)$ approximately $50 \%$ increase in protein-bound ClTyr content within recovered peritoneal lavage fluid proteins (Figure 2A). A dose-dependent effect of increased PON1 leading to reduced MPO activity, as monitored by ClTyr formation, was further supported by demonstration of an approximately $50 \%$ reduction $(P<0.01)$ in peritoneal lavage protein ClTyr content in PON1 Tg mice relative to the WT controls (Figure 2A). Thus, PON1 shows a dose-dependent inhibitory

\section{Figure 2}

MPO and PON1 inhibit each other's activity in vivo. (A) PON1 KO, WT, or PON1 Tg animals were injected i.p. with normal saline (BI) or thioglycollate broth $(T)$. Twenty-four hours later, peritoneal lavages were performed. Where indicated, mice were injected with zymosan (Z) 24 hours after thioglycollate, and peritoneal lavage was performed 4 hours later. Chlorotyrosine (CITyr) content (normalized to the precursor amino acid tyrosine [Tyr]) in soluble proteins recovered from peritoneal lavage was determined by stable isotope dilution LC/MS/MS analysis. (B) MPO KO, WT, or MPO Tg animals were injected i.p. with normal saline or zymosan (Z). After 72 hours of subacute peritonitis, serum was isolated and paraoxonase activity was measured.

effect on the production of the MPO-specific oxidation product, ClTyr, in an acute inflammation model.

In a parallel set of studies, we determined the impact of genetic alterations in MPO levels on systemic PON1 activity measured during inflammation. Here, serum paraoxonase activity was measured in a subacute peritonitis model following i.p. zymosan injection. In WT mice, chronic inflammation (72 hours) induced by the i.p. zymosan resulted in a significant $(P<0.05) 30 \%$ reduction in serum paraoxonase activity (Figure $2 \mathrm{~B}$ ). The initiation of peritonitis in the human MPO Tg mouse revealed an even more pronounced inhibition in systemic paraoxonase activity $(55 \%$, $P<0.001$ ), whereas the induction of peritonitis under comparable conditions within the MPO KO mouse failed to change systemic PON1 activity levels (Figure 2B). Importantly, the examination of circulating PON1 concentrations shows similar levels between MPO KO and MPO Tg mice (Supplemental Figure 3). Thus, changes observed in PON1 activity within serum are consistent with the inhibition of catalytic activity (specific activity) of the protein via an MPO-dependent process.

Hydrogen deuterium exchange mass spectrometry and mutagenesis studies reveal functional interaction sites on APOA1 of HDL and PON1. We next sought to identify sites of contact between PON1 and APOA1 of reconstituted nascent HDL (nHDL). Hydrogen-deuterium exchange mass spectrometry (HDX) is a powerful tool for identifying protein-protein interaction sites and was used to identify the MPO binding site on APOA1 of nHDL (23). We therefore used HDX to interrogate the potential site(s) on APOA1 within nHDL involved in PON1 interactions. Examination of amide proton deuterium incorporation factors in peptic peptides generated from APOA1 of nHDL in the absence or presence of PON1 revealed 2 regions of the APOA1 polypeptide chain with a significant $(>10 \%)$ reduction in deuterium incorporation factor in the presence of PON1. Multiple overlapping peptic peptides helped to define the regions showing a reduction in deuterium exchange in the presence of PON1, $\mathrm{Leu}_{38}-\mathrm{Leu}_{46}$ (termed P1, located at the boundary between the APOA1 $\mathrm{N}$-terminal and helix 1 ) and $\mathrm{Thr}_{202}-$ $\operatorname{Arg}_{215}$ (termed P2, located at the boundary between helix 8 [7 residues] and helix 9 [7 residues]) (Figure 3, A and B). Examination of the locations of these regions on the recently proposed double superhelix model of nHDL developed using a combination of contrast variation, small-angle neutron scattering, HDX, and chemical cross-linking/mass spectrometric studies (37) revealed that P1 and P2 are predicted to reside directly opposite to one another on the 2 antiparallel APOA1 chains (Figure 3C). Moreover, they are in close spatial proximity to the site of interaction previously identified between MPO and APOA1 in NHDL (APOA1 residues 190-203) (23). Of note, the identified peptide regions P1 and P2 of APOA1 
A

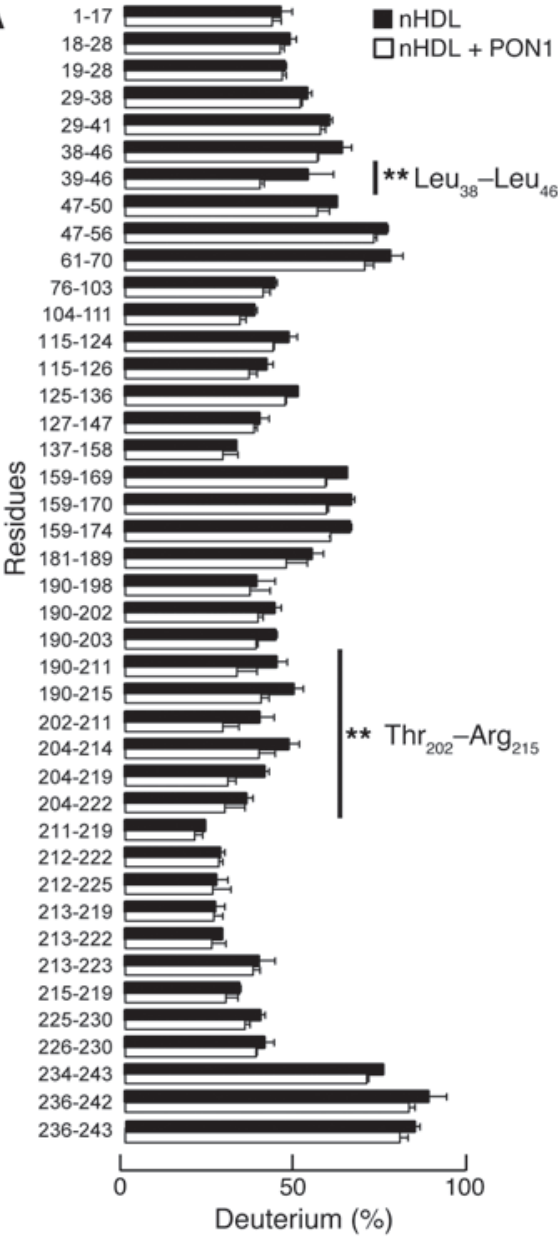

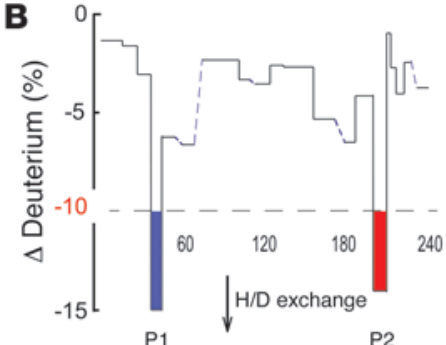

$P 1_{\text {WT }}$ L $_{38}$ GKQLNLKL $_{46} \quad P 2_{\text {WT }} \mathrm{S}_{201}$ TLSEKAK $_{208}$ $P 1_{\text {mut }} L_{38}$ GEALALEL $_{46} \quad P 2_{\text {mut }} S_{201}$ ALAAEAE $_{208}$

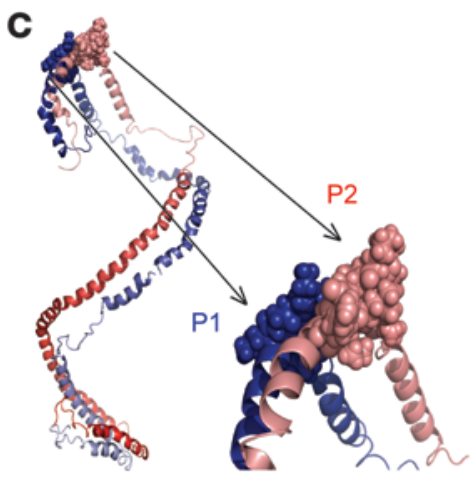

\section{Figure 3}

PON1 interaction sites on APOA1 within $\mathrm{nHDL}$ revealed by HDX MS. (A) Plot indicating the percentage of deuterium incorporation of exchangeable protons within individual peptic peptides of APOA1 in $\mathrm{nHDL}$ in the absence (black bars) versus presence (white bars) of PON1. ${ }^{*} P<0.05$. (B) Plot showing percentage change in deuterium incorporation of individual peptic peptides of APOA1 in $\mathrm{nHDL}$ in the absence or presence of PON1. Two regions of APOA1 demonstrate a significant reduction in deuterium incorporation in the presence or absence of PON1/P1 ( $\mathrm{L}_{38} \mathrm{GKQL}$ $\left.\mathrm{NLKL}_{46}\right)$ and P2 $\left(\mathrm{S}_{201} \mathrm{TLSEKAK}_{208}\right)$. Dotted line marks the $10 \%$ reduction in deuterium incorporation. (C) Illustration of PON1 interaction domains $\mathrm{P} 1$ and P2 on APOA1 within the double superhelix model of nHDL. The two APOA1 molecules are aligned in a head-to-tail antiparallel arrangement using a helix $5 /$ helix 5 registry. $\mathrm{N}$-termini are shown in dark red/blue, and $\mathrm{C}$-termini are shown in light red/blue. PON1 interaction sites on $\mathrm{nHDL}$ (P1, blue; P2, light red) are located directly opposite or adjacent to one another on the antiparallel APOA1 chains in every reported HDL model available as a Protein Data Bank (PDB) file (37-45).

To examine the functional significance of the APOA1 sequences identified as potential contact sites between nHDL and PON1, site-directed mutant forms of APOA1 were generated, whereby the WT sequences within P1 $\left(\mathrm{L}_{38}\right.$ GKQLNLKL 46$)$ and P2 $\left(\mathrm{S}_{201}\right.$ TLSE$\mathrm{KAK}_{208}$ ) were altered, removing potential hydrogen bond and ionic interaction sites $\left(\mathrm{P} 1_{\text {mut }}=\mathrm{L}_{38} \mathrm{GEALALEL}_{46}\right.$ and $\mathrm{P} 2_{\text {mut }}=$ $\mathrm{S}_{201}$ ALAAEAE $_{208}$ ) (Figure 3B). Initial control studies using recombinant APOA1 mutant forms generated with either the $\mathrm{P} 1_{\text {mut }}$ or $\mathrm{P} 2$ mut sequences revealed that both were functionally active and indistinguishable from human APOA1 with respect to overall structure as monitored by circular dichroism (Supplemental Figure 4A) and ABCA1-dependent efflux activity from cholesterol-loaded RAW cells (Supplemental Figure 5A). Similarly, nHDL produced using recombinant human APOA1 (WT) versus either the $\mathrm{P} 1_{\text {mut }}-$ or $\mathrm{P} 2_{\text {mut }}$-containing APOA 1 forms showed similar overall structure (Supplemental Figure 4B) and comparable total cholesterol efflux activity with cholesterol-loaded RAW cells (Supplemental Figure 5B). Surface plasmon resonance spectroscopy was also performed to evaluate the binding interaction changes between PON1 and nHDL produced using recombinant human APOA1 (WT) versus either the $\mathrm{P} 1_{\text {mut }^{-}}$or $\mathrm{P} 2_{\text {mut }}$-containing APOA1 mutant forms. $K_{\mathrm{D}}$ values of interaction between PON1 and $\mathrm{nHDL}$ formed with either the $\mathrm{P} 1_{\text {mut }}$ or $\mathrm{P} 2_{\text {mut }}$ recombinant $\mathrm{APOA} 1$ forms were decreased by 1.6-fold and 2.6-fold, respectively, compared with the observed $K_{\mathrm{D}}$ values for interaction of PON1 with $\mathrm{nHDL}$ formed with recombinant human (WT) APOA1 (Supplemental Figure 6). We therefore next examined whether $\mathrm{P} 1$ or $\mathrm{P} 2$ sequences in APOA1 were involved in PON1 interactions. Purified PON1 is more thermally labile in the absence of HDL and can lose catalytic activity with prolonged incubation at $37^{\circ} \mathrm{C}$ (Figure 4A). However, in the presence of either lipid-free APOA1 (Figure 4A, green curve), phospholipid vesicles (Figure 4B, purple curve), or nHDL (Figure $4 \mathrm{~B}$, green curve), PON1 activity is both stimulated and stabilized over time, consistent with binding to both lipid and protein components of reconstituted nHDL. Remarkably, incubation of PON1 with either of the $\mathrm{P} 1_{\text {mut }}$ - or $\mathrm{P} 2_{\text {mut }}$-containing recombinant APOA1 forms or the reconstituted nHDL generated using these mutant APOA1 forms showed intermediate capacity to bind, stimulate, and stabilize PON1 activity during prolonged incubations at $37^{\circ} \mathrm{C}$ (Figure 4, A and B). The intermediate phenotypes (protection from thermal denaturation) observed with nHDL formed using the mutant P1 or P2 sequences is consistent with these regions on APOA1 serving a functional role in partially supporting the HDLPON1 interaction. In another series of experiments, we sought to determine whether the strength of the HDL-PON1 binary complex influences MPO activity. Of note, while the binary complex formed by PON1 with nHDL that had been formed using WT 


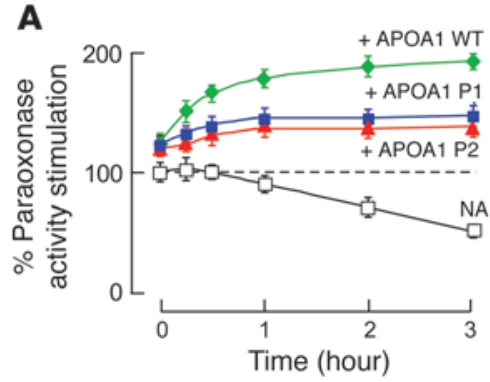

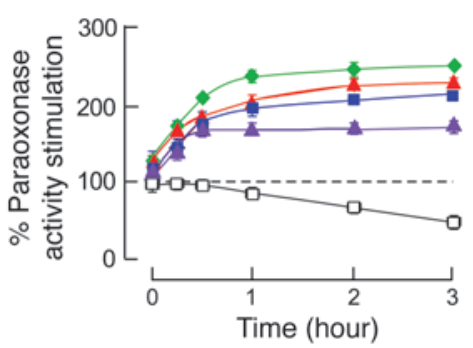

C

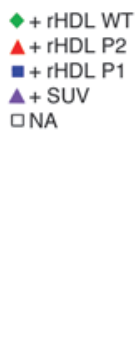

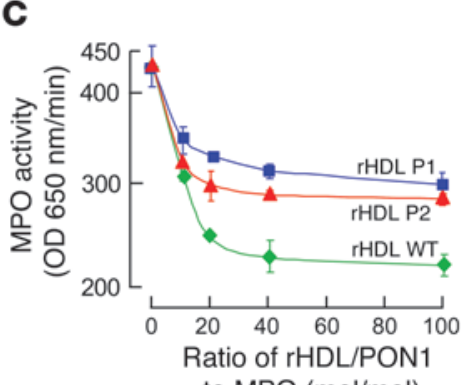

to $\mathrm{MPO}(\mathrm{mol} / \mathrm{mol})$

\section{Figure 4}

Demonstration that APOA1 P1 and P2 regions are functionally important in the HDL-PON1-MPO ternary complex. (A) APOA1 harboring mutations within $\mathrm{P} 1$ or $\mathrm{P} 2$ region (versus WT) were incubated with $\mathrm{PON} 1$ at $37^{\circ} \mathrm{C}$ for the indicated times, and then paraoxonase activity was determined. Results were normalized (100\%) to PON1 activity measured with no addition (NA) at $t=0$. (B) PON1 was incubated with reconstituted HDL (rHDL; 100:10:1, OPC/cholesterol/APOA1, $\mathrm{mol} / \mathrm{mol} / \mathrm{mol}$ ) using the indicated APOA1 forms (WT versus P1 versus P2 mutants) or small unilamellar vesicles (SUV) composed of 1-palmitoyl-2-oleoyl-sn-glycero-3-phosphocholine (POPC) (16:0, 18:1 phosphatidylcholine [PC]) for the indicated times, and then paraoxonase activity was measured. Results were normalized (100\%) to PON1 activity measured with no addition at $t=0$. (C) TMB assay showed the effect of varying levels of the binary complex composed of PON1 and the indicated rHDL (made with WT versus $\mathrm{P} 1$ versus the P2 APOA1 mutant) on MPO activity. All results represent the mean \pm SD from at least 3 independent experiments. In $\mathbf{A}$ and $\mathbf{B}$, the differences between WT and mutants at the 3 -hour point were significant (APOA1 WT versus APOA1 P1, $P<0.05$; APOA1 WT versus APOA1 $\mathrm{P} 2, P<0.05)$. In $\mathrm{C}$, the difference between WT and mutants at a molar ratio of rHDL/PON1 to MPO of 20 or greater were significant (rHDL WT versus rHDL $\mathrm{P} 1, P<0.05$; rHDL WT versus rHDL P2, $P<0.05)$.

recombinant human APOA1 inhibited MPO activity by approximately 50\%, the PON1 complex formed with nHDL prepared with either the P1 or P2 APOA1 mutant demonstrated a reduced ability to inhibit MPO activity (Figure 4C).

PON1 Tyr $r_{71}$ is a functionally important site for HDL interaction and a target for MPO-catalyzed oxidation in human atherosclerotic plaque. To investigate sites on PON1 that may participate in HDL interactions, we generated reconstituted nHDL, incorporating instead of cholesterol, a synthetic photoactivatable cholesterol analog containing a diazirine moiety at $\mathrm{C} 6$ of the sterol, as described in Methods (Figure 5A). Following exposure to UV light, the carbene diradical formed by $\mathrm{N} 2$ elimination from the diazirine has an exceedingly short half-life, with diffusion-limited reactivity with water (46). Thus, in the presence of an HDL binding partner such as PON1, UV light exposure is anticipated to produce a covalent adduct only with PON1 residues in close enough spatial proximity to the lipid phase of nHDL to exclude water (Figure 5A). Following coincubation of PON1 and nHDL and brief UV light exposure, mass spectrometry identified only one residue within PON1 that produced a cholestanyl adduct: $\mathrm{Tyr}_{71}$ (Figure 5, A and $\mathrm{B})$. Remarkably, separate in vitro studies incubating HDLPON1 $(1: 1, \mathrm{~mol} / \mathrm{mol})$ complexes with either the MPO $/ \mathrm{H}_{2} \mathrm{O}_{2} /$ $\mathrm{Cl}^{-}$system or the $\mathrm{MPO} / \mathrm{H}_{2} \mathrm{O}_{2} / \mathrm{NO}_{2}{ }^{-}$system at low oxidant levels $\left(\mathrm{H}_{2} \mathrm{O}_{2} /\right.$ APOA1, 10:1, $\left.\mathrm{mol} / \mathrm{mol}\right)$ identified in a reproducibly similar manner $\mathrm{Tyr}_{71}$ of PON1 as a preferred site of MPO-catalyzed oxidative modification, whereby $\mathrm{Tyr}_{71}$ of PON1 was detected as either a 3-chlorotyrosine or 3-nitrotyrosine adduct within tryptic peptides by proteomics studies (Supplemental Figures 7, A and B). The crystal structure of PON1 was recently reported (47, 48), and PON1 $\mathrm{Tyr}_{71}$ sits near the putative interfacial region of the hypothetical HDL binding domain (Figure 5C), suggesting both that $\mathrm{Tyr}_{71}$ of PON1 may support HDL interaction, and that MPO-dependent site-specific oxidative modification of PON1 at $\mathrm{Tyr}_{71}$ may impair PON1 function in vivo. The potential physiological relevance of PON1 Tyr 71 oxidation by MPO was further supported by proteomics studies of PON1 recovered from human atherosclerotic plaque, in which $\mathrm{ClTyr}_{71}$ was directly visualized on PON1 tryptic peptides (Supplemental Figure 8). These results demonstrate that within the proinflammatory environment of the human atherosclerotic plaque where site-specific oxidation of APOA1 through both nitration and chlorination has been shown to occur by MPO $(6,23,35)$, the HDL-associated protein PON1 too is oxidized by the heme protein.

In an additional series of studies, we sought to further explore the potential functional significance of posttranslational oxidative modification of $\mathrm{Tyr}_{71}$ of PON1. Site-directed mutagenesis studies of recombinant human PON1 were performed, whereby tyrosine was converted to multiple alternative amino acids (alanine, aspartic acid, or lysine). Each of the recombinant mutant PON1 forms and recombinant human PON1 were next individually incubated with nHDL $(1: 1, \mathrm{HDL}-\mathrm{PON} 1, \mathrm{~mol} / \mathrm{mol})$ at $37^{\circ} \mathrm{C}$, and then residual PON1 catalytic activity was quantified. Functional analyses of recombinant human PON1 versus the site-directed mutant forms of PON1 (aka Tyr ${ }_{71}$ Ala, $\mathrm{Tyr}_{71}$ Asp, or $\mathrm{Tyr}_{71} \mathrm{Lys}$ ) showed that in each case, the mutant PON1 form had markedly reduced catalytic activity (Figure 5D). Collectively, these studies show that $\mathrm{Tyr}_{71}$ of PON1 is both a site-specific target for MPO-catalyzed oxidation in human atherosclerotic plaque and a functionally important residue for HDL interaction and preservation of PON1 activity.

HDL-bound PON1 is a selective target for MPO-catalyzed oxidation and functional impairment during cardiovascular disease. We next sought to further examine the functional and potential clinical significance of the interaction between MPO and PON1 within the HDL-PON1-MPO ternary complex during cardiovascular disease. Subjects $(n=26)$ presenting for diagnostic coronary angiography at the time of ACS were consented, and blood was drawn for systemic PON1 activity determination and HDL isolation and chlorotyrosine content determination. In parallel, age-, sex-, and blood pressure-matched healthy nondiabetic control subjects $(n=26)$ were consented and similarly examined. Baseline charac- 
A
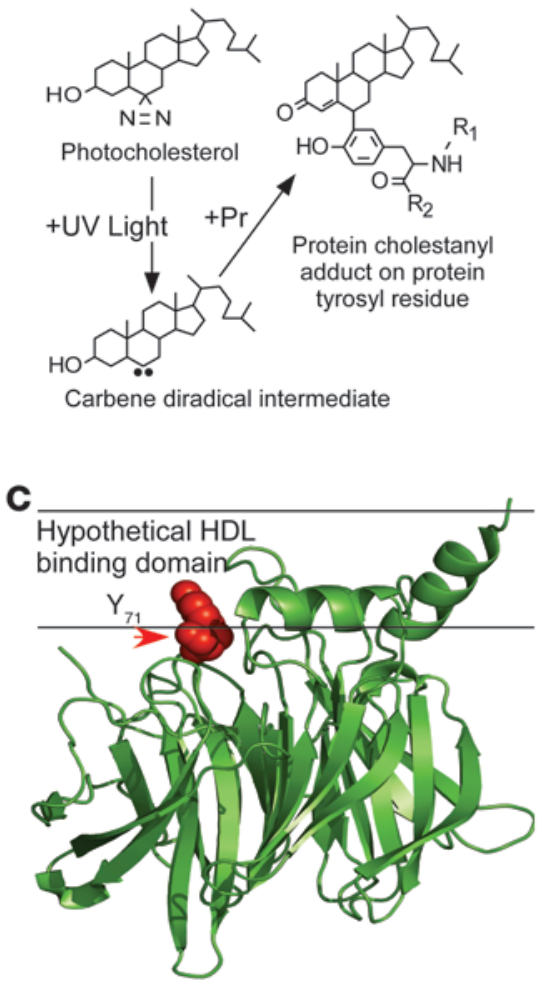

B
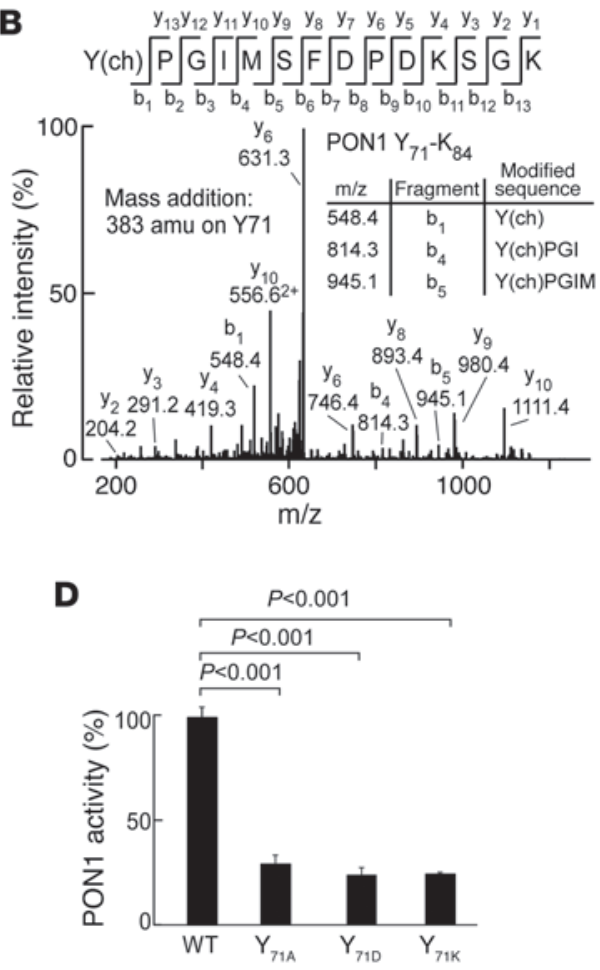

\section{Figure 5}

PON1 $\mathrm{Tyr}_{71}$ is a functionally important site for HDL interaction and a target for MPOcatalyzed oxidation in human atherosclerotic plaque. (A) Schematic illustration of photocholestanyl-labeled PON1 tyrosyl residue. (B) Reconstituted HDL formed using POPC/ photocholesterol/APOA1 (100:10:1) was incubated with PON1 and exposed to UV light as described in Methods. Proteomics studies revealed the photocholestanyl adduct of PON1 $\mathrm{Tyr}_{71}[\mathrm{Y}(\mathrm{ch})]$ as a site on PON1 that directly interacts with cholesterol as described in Methods. $\mathrm{m} / \mathrm{z}$, mass-to-charge ratio. (C) PON1 crystal structure with superimposed location of $\mathrm{Tyr}_{71}$ (red) and hypothetical HDL binding surface based on hydrophobicity analysis. (D) Recombinant human WT PON1 versus the indicated PON1 $\mathrm{Tyr}_{71}$ site-specific mutants were generated, isolated, incubated with HDL, and then paraoxonase activity was determined as described in Methods. Results represent the mean \pm SD from at least 3 independent experiments. teristics and laboratory analyses within the case-control cohort are shown in Supplemental Table 1, and Figure 6A shows a scatter plot demonstrating the relationship between systemic PON1 activity measured within each group and protein-bound 3-chlorotyrosine content in the isolated HDL as determined by stable isotope dilution LC/MS/MS. Of note, examination of the relationship between the chlorotyrosine content of HDL and systemic PON1 activity showed a significant inverse association within the combined ACS plus healthy control cohort $(r=-0.54, P<0.001)$. Further, despite substantial overlap, systemic PON1 activity was significantly $(P<0.05)$ reduced within the ACS cohort compared with the healthy controls (Figure 6A), and protein-bound chlorotyrosine content of isolated HDL, a specific molecular marker of MPOcatalyzed oxidation $(29,31)$, was also elevated $(P<0.01)$ within the ACS cohort relative to the healthy controls (Figure 6A). The small size of this study, however, prevents examination of the impact of comorbidities on these associations.

In a final series of studies, we further investigated the oxidative inactivation of PON1 during ACS. A separate case-control study was performed for more detailed proteomics analyses involving an independent cohort of ACS subjects $(n=10)$ and age-, sex-, and blood pressure-matched healthy nondiabetic controls $(n=10)$. Baseline characteristics and laboratory analyses within this independent case-control cohort are shown in Supplemental Table 2. HDL was isolated from subjects, and then PON1 tryptic peptides were examined by proteomics. Methionine residues are highly susceptible to oxidation and conversion into methionine sulfoxide. We therefore quantified site-specific methionine residue oxidation within PON1 recovered from the isolated HDL in both ACS and control subjects, as described in Methods. Multiple methionine residues within PON1 were detected, and 3 (Met ${ }_{88}$, Met ${ }_{55}$, and
Met $_{12}$ ) were reproducibly observed, permitting quantification of both parent (unmodified) and oxidized forms (exemplary spectra shown in Supplemental Figures 9 and 10) within all samples. Interestingly, the levels of oxidized $\mathrm{Met}_{88}$ were significantly elevated $(P<0.05)$ within the PON1 recovered from ACS subjects relative to controls (Figure 6B and Supplemental Figure 11), indicating that PON1 undergoes site-specific oxidative modification during ACS. To assess whether methionine oxidation may have functional significance for PON1 activity, we examined the impact of methionine sulfoxide reductase incubation with PON1 that had previously been partially functionally inactivated by exposure to the $\mathrm{MPO} / \mathrm{H}_{2} \mathrm{O}_{2} / \mathrm{Cl}^{-}$system. Remarkably, incubation of oxidized PON1 with methionine sulfoxide reductase significantly reversed a portion of the PON1 inactivation induced by MPO-generated oxidants (Figure 6C).

\section{Discussion}

MPO release from activated leukocytes, binding to HDL, and targeting of APOA1 for oxidative modification and functional inactivation of the particle are phenomena recognized to occur during inflammation and have been linked to the generation of "dysfunctional" forms of $\operatorname{HDL}(6,23,24,35,49-51)$. Moreover, a reduction in systemic PON1 activity during cardiovascular disease in mice and humans has been reported and has been linked to the loss of antiinflammatory properties of HDL isolated from ACS subjects $(7,15,19)$. Finally, a role for MPO in the initiation of lipid peroxidation in vivo $(33,34)$ and a reciprocal association between PON1 activity and systemic measures of oxidant stress in mice and humans are well documented under a variety of inflammatory conditions $(15,19,20)$. That these functions of MPO and PON1, both HDL-associated proteins, should be mechanistically 
A

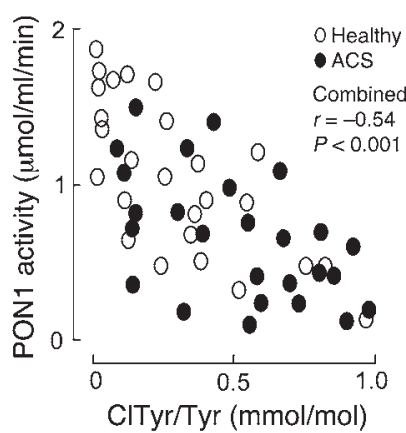

B

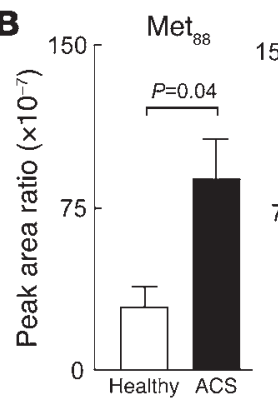

C

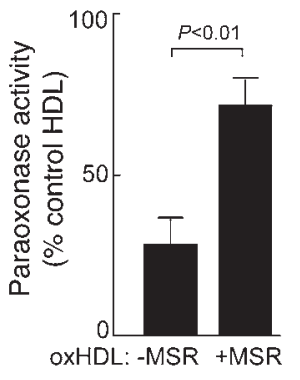

\section{Figure 6}

Methionine oxidation plays a role in PON1 activity and stability. (A) Inverse correlation between systemic PON1 activity and protein content of CITyr-isolated HDL from ACS (black ovals; $n=26$ ) and healthy nondiabetic control subjects (white ovals; $n=26$ ) from case-control cohort 1 (see Supplemental Table 1). $P$ value shown is for the Spearman's rank correlation between PON1 activity level and HDL CITyr content among both control and ACS subjects. (B) Quantification of site-specific methionine oxidation (methionine sulfoxide) within PON1 recovered from isolated HDL from ACS $(n=10)$ and healthy nondiabetic control subjects $(n=10)$ enrolled in case-control cohort 2 (Supplemental Table 2). Results shown are expressed as the peak ratio of peptide harboring the indicated methionine sulfoxide residue or parent (methionine-harboring) peptide relative to the reference peptide, as described in Methods. (C) Isolated human HDL from a healthy donor was incubated with the MPO/ $\mathrm{H}_{2} \mathrm{O}_{2} / \mathrm{Cl}^{-}$system (oxHDL), and then the effect of exposure to either methionine sulfoxide reductase (+MSR) or vehicle control (-MSR) on PON1 activity was determined as described in Methods. PON1 activity is expressed relative to paraoxonase activity measured in $\mathrm{HDL}$ prior to exposure to the $\mathrm{MPO} / \mathrm{H}_{2} \mathrm{O}_{2} / \mathrm{Cl}^{-}$system. Data are the mean $\pm \mathrm{SD}$ of triplicate determinations.

linked has not yet been examined. We believe that the present studies demonstrate for the first time both the reciprocal biochemical functional relationship between MPO and PON1 activities as they relate to systemic measures of oxidant stress and HDL function and a structural basis accounting for the linkage of these phenomena - namely, that MPO, PON1, and HDL can form a ternary complex whereupon each member of the complex interacts with and functionally impacts the other's activity in vivo. Thus, the present studies show that like APOA1 $(6,23)$, the HDL-associated protein PON1 may be targeted for oxidative modification and functional inactivation by MPO in vivo, as observed using both animal models of inflammation and in human clinical studies of the acute inflammatory condition, ACS.

A model of a hypothetical ternary complex of MPO and PON1 bound to nHDL is illustrated in Figure 7 and can help to provide a structural rationale for the observed reciprocal regulatory behavior among MPO, PON1, and HDL within the context of the present studies and prior reported observations. The model is not intended to represent a true all-atom structure, but rather is an illustration providing a structural and mechanistic rationale upon which a variety of biophysical and functional observations can be explained. The model shown is based on the known crystal structures of PON1 (48) and MPO (52) and represents a model of HDL based on small-angle neutron scattering and HDX studies of nHDL (37), prior reported sites of MPO-catalyzed oxidative modification to APOA1 in $\operatorname{HDL}(23,35,49,53)$, previously identified MPO-APOA1 interaction sites based on hydrogen/deuterium (H/D) exchange and functional studies (23), and newly discovered sites of oxidation or interaction among the various participants in the ternary complex from the present studies. Of note, while the discussions of the ternary complex below are based on the double superhelix model of nHDL (37) as the scaffolding upon which MPO and PON1 are bound (Figure 7), alternative models for the HDL-PON1-MPO ternary complex using either a discoidal HDL (e.g., solar flare model) (ref. 43 and Supplemental
Figure 12) or a spherical HDL model (ref. 44 and Supplemental Figure 13) as the lipoprotein scaffolding show similar conceptual findings in terms of distance constraints between identified contact sites within the complex and provide a rationale for sites of MPO-catalyzed oxidation observed on APOA1 and PON1 in vitro and in vivo. Because the double-belt discoidal model of HDL contains a truncated APOA1 (residues 44-243), which misses 6 residues from the P1 binding site, it was not included. The model of the ternary complex is based on the nonoverlapping but directly adjacent sites on APOA1 of nHDL to which the interaction sites with MPO and PON1 map. The close proximity of these HDL-associated proteins on the HDL scaffold, coupled with the high affinity MPO and PON1 show for one another, suggest that there may be some direct interaction between PON1 and MPO when they are both associated with HDL, and this appears to be at the origin of the mutual activity modulation. This observation is supported by the data in Figure 1C, which clearly indicate that MPO activity, when it associates with PON1 either alone or on an HDL particle, is about half of its activity compared with the absence of PON1.

PON1 is an HDL-associated enzyme under consideration as a potential therapeutic for cardiovascular disease. While studies in both mice $(19,20)$ and humans $(15)$ support an antioxidant function for the protein, the precise mechanism(s) through which PON1 promotes its systemic antiinflammatory and antioxidant effects are not well defined. Moreover, systemic PON1 activity levels can vary over a very broad range (orders of magnitude), while PON1 mass shows less variation, with PON1 abundance in the circulation being approximately one-tenth that of HDL particles (36). The mechanism(s) contributing to the wide variation in systemic PON1 activity observed between subjects are not well known, but early in vitro studies established the sensitivity of PON1 to chemical oxidants (54). The present studies show that under inflammatory conditions, MPO-mediated oxidative inactivation contributes to modulation of PON1 in vivo. It should 


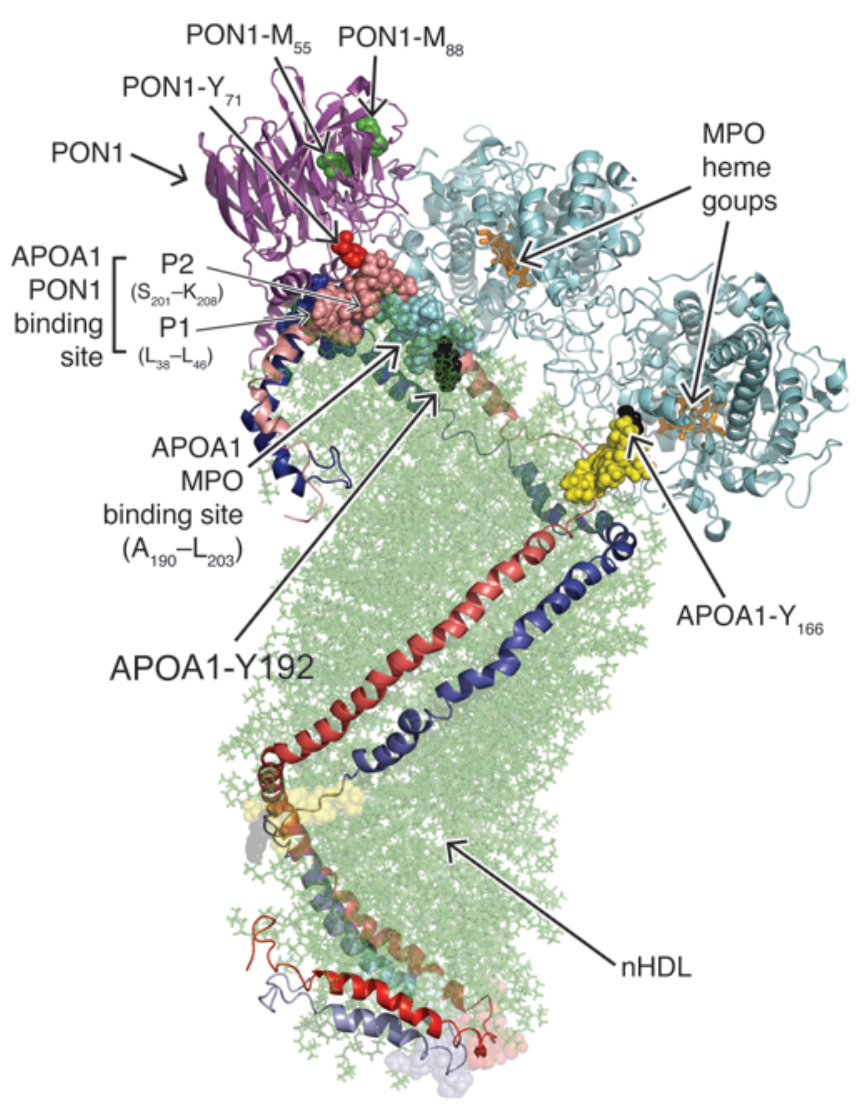

be noted that functional polymorphisms can also contribute to variations in PON1 activity, but the magnitude of alterations are relatively modest compared with the breadth of PON1 activity levels observed in the systemic circulation $(15,55)$.

Despite the interest in PON1 and HDL as potential therapeutic agents, the structural basis of the PON1-HDL interface has not yet been defined. Our studies show that PON1 binding to HDL is important for the maintenance of PON1 activity, with both lipid and protein components of HDL supporting PON1 interaction (Figure 4). Further, using a combination of novel biophysical approaches, coupled with site-directed mutagenesis and subsequent functional studies, both $\mathrm{Tyr}_{71}$ of PON1 and the APOA1 sequences P1 ( $\left.\mathrm{L}_{38} \mathrm{GKQLNLKL}_{46}\right)$ and P2 $\left(\mathrm{S}_{201} \mathrm{TLSEKAK}_{208}\right)$ were identified as critical residues in supporting HDL-PON1 interaction (Figures 3-6). PON1 $\mathrm{Tyr}_{71}$ was unambiguously identified as a contact site with the lipid phase of HDL through the formation of a cholestanyl adduct using a reconstituted nHDL particle generated with a novel photoactivatable cholesterol analog (Figure 5). The carbene diradical formed on the cholesterol analog following UV irradiation reacts at a near-diffusion-limited rate with $\mathrm{H}_{2} \mathrm{O}$, indicating that the interaction between the HDL lipid phase and PON1 $\mathrm{Tyr}_{71}$ is sufficiently tight to exclude water, and suggesting that the phenol moiety of $\mathrm{Tyr}_{71}$ extends into the lipid phase upon HDL binding (Figure 8). It is remarkable that recent crystallographic studies of PON1 in a ligand-bound form identify PON1 $\mathrm{Tyr}_{71}$ as a critical residue that undergoes conformational change upon ligand binding and plays an important role in a hydrogen bond network near the active site (Figure 8 and ref. 48). Our studies confirm the functional significance of

\section{Figure 7}

Structural model of a hypothetical ternary complex of MPO and PON1 bound to $\mathrm{nHDL}$. Illustration of an HDL-MPO-PON1 ternary complex composed of the double superhelix model of $\mathrm{nHDL}$ and the crystal structures of MPO and PON1 bound to one another with identified protein-protein interaction sites. The two predominantly $\alpha$ helical APOA1 chains in $\mathrm{nHDL}$ are aligned in a head-to-tail antiparallel arrangement. $\mathrm{N}$-termini are shown in dark red/blue, and C-termini are shown in light $\mathrm{red} / \mathrm{blue}$. Phospholipids in the lipid core of $\mathrm{nHDL}$ are depicted in semitransparent green. PON1 binding sites on APOA1 shown are $\mathrm{P} 1\left(\mathrm{~L}_{38^{-}}\right.$ $\mathrm{L}_{46}$, solid blue) and $\mathrm{P} 2\left(\mathrm{~S}_{201}-\mathrm{K}_{208}\right.$, solid light red). Adjacent MPO binding site on APOA1 (residues $A_{190}-L_{203}$, filled light blue) is also depicted. Site-specific oxidative modifications found in PON1 recovered from isolated HDL from either atherosclerotic lesions or ACS plasma include $\mathrm{Tyr}_{71}$ (red) and $\mathrm{Met}_{55}$ and Met 88 (both green). Met 12 of PON1 is not shown because the $\mathrm{N}$ terminus (16 amino acids) of PON1 was not resolved in the PON1 crystal structure reported. Location of the openings to the two heme pockets on the MPO homodimer are predicted to be in close spatial proximity to the site-specific oxidative modifications reported in APOA1 recovered from human atherosclerotic plaque, $\mathrm{Tyr}_{166}$, and $\mathrm{Tyr}_{192}$ (solid black). Also shown are the "solar flare" regions of APOA1, presumed LCAT interaction sites (solid yellow).

PON1 Tyr $_{71}$ for HDL interaction through site-directed mutagenesis, since multiple PON1 mutants lacking $\mathrm{Tyr}_{71}$ demonstrated reduced HDL binding, thermal stability, and enzymatic activity (Figures 5 and 6). Finally, the in vivo significance of this residue is further suggested both by the demonstration that it is targeted for MPO-catalyzed nitration and chlorination in model systems under physiological levels of oxidant (Supplemental Figure 7) and by the detection of PON1 3-chloroTyr $\mathrm{T}_{71}$, a specific posttranslational modification formed by MPO in vivo, in PON1 recovered from human atherosclerotic plaque (Supplemental Figure 8). Importantly, examination of the crystal structure of PON1 indicates that posttranslational modification of the phenolic ring of $\mathrm{Tyr}_{71}$ with a bulky substituent (e.g., $\mathrm{NO}_{2}, \mathrm{Cl}$ ) will also interfere sterically with the active site cavity walls and prevent $\mathrm{Tyr}_{71}$ from forming a hydrogen bond with PON1 Asp 183 (Figure 8), a critical residue that modulates access to the PON1 active site (48). While the site of PON1 interaction with HDL was previously unknown, the recent crystal structures reported for both free and ligand-bound forms of PON1 suggest a possible lipid interfacial binding surface based on predicted hydrophobicity characteristics of the protein $(47,48)$. It is notable that PON1 $\mathrm{Tyr}_{71}$ is structurally positioned near the hypothesized lipid-binding domain within PON1 (Figure 5).

The present studies also help explain, in part, the specificity of PON1 for interaction with HDL over alternative hydrophobic surfaces and lipoproteins, since specific docking sequences on APOA1 were also identified, showing functional importance in HDLPON1 interaction. HDX mass spectrometric studies revealed that 2 APOA1 regions $\left(\mathrm{L}_{38}-\mathrm{L}_{46}\right.$ and $\left.\mathrm{S}_{201}-\mathrm{K}_{208}\right)$ display substantial reduc- 

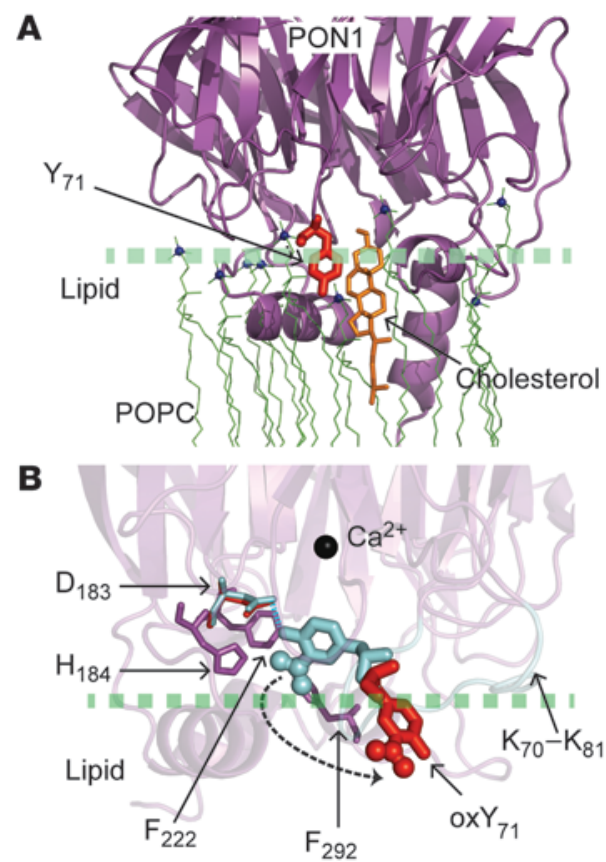

tions in deuterium incorporation at the amide proton in the presence versus absence of PON1, suggesting within the nHDL particle that APOA1 binding to PON1 in these regions is tight enough to partially exclude $\mathrm{D}_{2} \mathrm{O}$ (Figure 3 ). It should be noted, however, that an alternative explanation for the $\mathrm{H} / \mathrm{D}$ exchange data might be that upon PON1 binding, APOA1 within HDL undergoes conformational changes that reduce amide proton exchange rates at these residues. Mutagenesis studies, however, were also consistent with the findings that these regions on APOA1 play a direct role in PON1-HDL interaction, since these studies confirmed that both $\mathrm{P} 1$ and $\mathrm{P} 2$ sequences are functionally important in PON1 binding to HDL and in the maintenance of PON1 activity (Figures 3 and 4). These APOA1 residues, however, do not appear to be critical for either HDL formation or cholesterol efflux function (Supplemental Figures 4 and 5). Notably, what was found upon examination of the anatomic location of the two APOA1 regions (P1 and P2) on nHDL that help support binding to PON1 provides a logical explanation for why these two apparently distant regions on the APOA1 polypeptide chain support PON1 interaction - they are predicted to be directly adjacent to one another on the two antiparallel APOA1 chains within HDL (Figures 3 and 7, and Supplemental Figures 12 and 13).

Perhaps one of the more remarkable findings in the present studies is the previously unrecognized reciprocal functional interaction between MPO and PON1. In vitro studies with both isolated MPO and PON1 in serum or activated neutrophils from normal and MPO-deficient subjects demonstrate reciprocal effects on PON1 and MPO activity within serum (Figure 1), and studies with PON1 KO and Tg mice or MPO KO or Tg mice confirmed the dose-dependent reciprocal effects of each protein on the other's enzymatic activity in vivo during inflammation (Figure 2). Finally, the H/D exchange studies demonstrate that the site on APOA1 supporting PON1 binding is in close spatial proximity to the previously identified MPO binding site on APOA1 of nHDL (23), and proteomics studies on PON1 reveal that $\mathrm{Tyr}_{71}$, along

\section{Figure 8}

Role of PON1 Tyr $r_{71}$ in HDL binding and catalysis. (A) Illustration indicating how $\mathrm{Ty}_{71}$ of PON1 may interact with the cholesteryl group (orange) of nHDL. PON1 crystal structure (PDB ID: 1V04) (purple) is shown docking with the lipid phase of nHDL (POPC molecules are shown as green lines, with the nitrogen atom of the choline group represented by blue spheres), and $\mathrm{Tyr}_{71}$ (red) protrudes into the lipid phase, interacting with cholesterol. (B) Illustration comparing the conformation of PON1 Tyr ${ }_{71}$ in the "closed" and "open" conformations of PON1 recently reported by Tawfik and colleagues (48). The presumed "lid" domain $\left(\mathrm{K}_{70}-\mathrm{K}_{81}\right.$, cyan) in PON1 that seems to control access to the PON1 active site was resolved in the PON1 crystal structure with a lactone analog bound to the active site (PDB ID: 3SRG; closed conformation) only. If $\mathrm{Tyr}_{71}$ is nitrated, the bulky $\mathrm{NO}_{2}$ group interferes sterically with some of the residues $\left(H_{184}, F_{222}, F_{292}\right)$ (purple) in the active site walls. The halide $\mathrm{Cl}$ has an even larger van der Waals radius and would show similar steric interference. with multiple PON1 methionine residues (e.g., $\mathrm{M}_{55}$ and $\mathrm{M}_{88}$ ), are predicted to be in close spatial proximity to 1 of the 2 heme pockets of MPO (Figure 7). Indeed, we hypothesize that the structural rationale for PON1-dependent inhibition in MPO activity observed in vitro, which was approximately $50 \%$ of total activity (Figure 1), likely arises because PON1 binding to MPO may block substrate access to 1 of the 2 heme pocket active sites on the MPO homodimer (Figure 7). It should be noted that the precise interaction sites on MPO for PON1 binding, however, remain to be determined. The protease-resistant nature of MPO, coupled with its high degree of glycation, hinders structural elucidation of the residue(s) on MPO where it interacts with PON1, even though the binding interaction shows a $K_{\mathrm{D}}$ in the low nanomolar range and is tight enough to permit cross immunoprecipitation studies to pull down MPO or PON1 along with APOA1 with antibodies against the alternative partners in the ternary complex (Supplemental Figures 1 and 2).

Prior reported studies demonstrating the selective enrichment of $\mathrm{NO}_{2}$ Tyr and ClTyr within APOA1 recovered from serum or within human atherosclerotic vessels suggest a possible (patho)physiologic association between APOA1 and MPO in vivo. More recent in vivo studies involving the infusion of MPO into mice demonstrated reduced macrophage-to-feces reverse cholesterol transport (50), also strongly supporting a possible (patho)physiologic association between APOA1 and MPO in vivo. Additionally, this result might also indicate a negative effect of MPO on PON1 activity in part through interaction with HDL as a trimeric MPO-PON1HDL complex. Since PON1 has been suggested to help promote some of the cholesterol efflux activity of HDL (56-58), the present demonstrations of MPO-dependent oxidative modification and inactivation of PON1 in vitro and in vivo may be considered an alternative mechanism for generating a "dysfunctional HDL" particle (or perhaps more precisely, a "dysfunctional HDL-associated proteome") during inflammation. It is of interest that a recently published study examining systemic MPO activity and 
various HDL-related activities, including cholesterol efflux activity, antioxidant function, and PON1 activity, among subjects with rheumatoid arthritis noted that higher plasma MPO activity was associated with worse HDL functional measures (59). In summary, HDL appears to serve as the scaffolding upon which functionally related proteins can assemble and interact, serving as a mechanism to "fine tune" biological functions. The evidence provided herein reveals the existence of an HDL-MPO-PON1 ternary complex in which each partner in the complex functions to reciprocally modulate the other's activity, influencing oxidant stress and lipid peroxidation during inflammation. The demonstration that these proteins form a functional ternary complex, the identification of key residues supporting these interactions, and the demonstration that these residues may be targeted for oxidative modification and inactivation during inflammation provide a structural basis that may partially help explain many of the observations reported in both past and the present studies at the cellular, animal model, and human clinical investigation levels. Given the complexity and heterogeneity of the associated proteins observed in HDL, a vast number of distinct functional clusters of proteins on distinct HDL assemblies likely exist (60), further adding to the functional complexities of this multifaceted lipoprotein.

\section{Methods}

\section{Materials}

$\mathrm{L}-\left[{ }^{13} \mathrm{C}_{6}\right]$ tyrosine and L- $\left[{ }^{13} \mathrm{C}_{9},{ }^{15} \mathrm{~N}_{1}\right]$ tyrosine were purchased from Cambridge Isotope Laboratories. RAW264.7 cells were obtained from ATCC. $\left[{ }^{3} \mathrm{H}\right] \mathrm{Cho}-$ lesterol was obtained from Amersham Biosciences. Human MPO was isolated and characterized as described (29). All other reagents were obtained from Sigma-Aldrich unless otherwise specified.

\section{General procedures}

Protein content was determined by the Markwell-modified Lowry protein assay (61) with BSA as a standard. The concentration of $\mathrm{H}_{2} \mathrm{O}_{2}$ was determined spectrophotometrically $\left(\varepsilon_{240}=39.4 \mathrm{M}^{-1} \cdot \mathrm{cm}^{-1}\right)$, and the concentration of $\mathrm{NaOCl}$ was determined spectrophotometrically $\left(\varepsilon_{292}=350 \mathrm{M}^{-1} \cdot \mathrm{cm}^{-1}\right)$. LDL and HDL were isolated by sequential ultracentrifugation (62). The plasmid (G3C9) for generating recombinant PON1 was provided by Dan S. Tawfik (The Weizmann Institute of Science, Rehovot, Israel) and purified as described (63). Protein-bound ClTyr was quantified as described by stable isotope dilution LC/MS/MS analyses using synthetic $3-\mathrm{Cl}-\left[{ }^{13} \mathrm{C}_{6}\right]$ tyrosine and $\mathrm{L}-\left[{ }^{13} \mathrm{C}_{9},{ }^{15} \mathrm{~N}_{1}\right]$ tyrosine as internal standards on an ABI SCIEX QTRAP 5500 mass spectrometer $(23,31)$. PON1 activity was measured using a paraoxon substrate on an open channel of an Architect ci8200 platform (Abbott Diagnostics), and the rate of generation of paranitrophenol was determined at $405 \mathrm{~nm}$ using an extinction coefficient of 17,000 $\mathrm{M}^{-1} \cdot \mathrm{cm}^{-1}(21,22)$. Activity was determined in reaction mixtures composed of $1.5 \mathrm{mM}$ paraoxon, $10 \mathrm{mM}$ Tris $\mathrm{HCl}$ ( $\mathrm{pH} 8.0$ ), $1 \mathrm{M}$ sodium chloride, and $2 \mathrm{mM}$ calcium chloride at $24^{\circ} \mathrm{C}$. Results were expressed as micromoles of paranitrophenol produced per minute per milliliter of serum. Peroxidase activity was measured spectrophotometrically at $650 \mathrm{~nm}$ by using a TMB substrate. Cholesterol efflux (ABCA1-dependent and total) activity of APOA1 and nHDL were determined as described (23).

\section{Clinical specimens}

Two clinical case-control cohorts of ACS subjects were enrolled for these studies. The first was comprised of subjects $(n=26)$ presenting for diagnostic coronary angiography at the time of ACS and healthy nondiabetic control subjects ( $n=26$; age-, sex-, and blood pressure-matched) presenting to a pre- ventive cardiology clinic for risk factor evaluation and recommendations. An independent cohort of ACS subjects $(n=10)$ were enrolled from the Cardiovascular Center, University Hospital Zurich at the time of presentation, along with 10 healthy volunteers (age-, sex-, and blood pressure-matched controls) responding to advertisements. HDL particles were isolated by buoyant density centrifugation from atherosclerotic lesions found in arterial tissues obtained at the time of vascular surgery. All tissues were immediately rinsed in ice-cold PBS supplemented with $2 \mathrm{mM}$ diethylenetriaminepentaacetic acid (DTPA) and immediately snap frozen in buffer $(65 \mathrm{mM}$ sodium phosphate [pH 7.4], $2 \mathrm{mM}$ DTPA, and $100 \mu \mathrm{M}$ butylated hydroxytoluene) with liquid $\mathrm{N}_{2}$ and stored under argon at $-80^{\circ} \mathrm{C}$ until analysis.

\section{Mouse models of inflammation}

Age- and sex-matched C57BL/6J PON1 KO (19), human PON1 Tg (20) (>10 generations backcrossed onto a C57BL/6J background) and C57BL/6J WT mice were used for acute peritonitis studies. Animals were injected i.p. with $1 \mathrm{ml}$ of $4 \%$ thioglycollate broth. Twenty hours after recruitment, the mice were injected with zymosan $(250 \mathrm{mg} / \mathrm{kg})$ (34). Peritoneal lavage was performed 4 hours later with PBS containing $100 \mu \mathrm{M}$ butylated hydroxytoluene and $100 \mu \mathrm{M} \mathrm{DTPA}$, aliquoted into cryovials, and overlaid with argon, immediately flash frozen in liquid nitrogen, and stored at $-80^{\circ} \mathrm{C}$ until analysis. Age- and sex-matched C57BL/6J MPO KO (>10 generations backcrossed onto C57Blk/6J), C57Blk/6J human MPO Tg mice, and WT mice were used for subacute peritonitis studies. Zymosan was suspended in sterile PBS to a final concentration of $7 \mathrm{mg} / \mathrm{ml}$ and injected i.p. at a final dose of $100 \mathrm{mg} / \mathrm{kg}$. Seventy-two hours later, blood was drawn from the mice. The control animals received i.p. injections of PBS.

\section{Mass spectrometry of neutrophil-and MPO-generated oxidation products of arachidonic acid and linoleic acid}

Human PMNs were isolated from whole blood obtained from healthy and MPO-deficient subjects as described (33) and used immediately for experiments. PMNs $\left(10^{6} / \mathrm{ml}\right)$ were incubated at $37^{\circ} \mathrm{C}$ with $50 \%(\mathrm{v} / \mathrm{v})$ human serum under air in phenol red-free HBSS supplemented with $100 \mu \mathrm{M}$ DTPA, activated where indicated in Figure 1D and Figure 1E by adding $200 \mathrm{nM}$ phorbol myristate acetate (PMA), and maintained in suspension by gentle mixing every 5 minutes. After 2 hours, the reactions were stopped by immersion in an ice/water bath, followed by centrifugation at $4{ }^{\circ} \mathrm{C}$ and the immediate addition of $50 \mu \mathrm{M}$ butylated hydroxytoluene and $300 \mathrm{nM}$ catalase to the supernatant. Lipid peroxidation products in the supernatant were then rapidly assayed as described below. Reactions with isolated MPO were performed at $37^{\circ} \mathrm{C}$ in sodium phosphate buffer (20 mM, pH 7.0) supplemented with $100 \mu \mathrm{M}$ DTPA using $65 \mathrm{nM}$ MPO and $100 \mu \mathrm{M} \mathrm{H}_{2} \mathrm{O}_{2}$ final (added over at least 4 aliquots every 15 minutes). Reactions were terminated as described above for the PMN studies. Deuterated internal standard, 12(S)-hydroxy-5,8,10,14-eicosatetraenoic-5,6,8,9,11,12,14,15-d8 acid (12-HETE-d8; Cayman Chemical Company) was added, lipids were extracted, and both precursors (arachidonic and linoleic acids) and oxidized fatty acids were quantified with stable isotope dilution LC/MS/MS using established methods on an ABI SCIEX API 5000 triple quadrupole mass spectrometer (34).

\section{Human APOA1 isolation and nHDL preparation}

Human APOA1 was purified from isolated human HDL, and recombinant mutant forms of human APOA1 were prepared, the His tag was removed, and the forms were isolated as described (43). Reconstituted nHDL was prepared using the modified sodium cholate dialysis method (64) at an initial molar ratio of 100:10:1 of POPC/cholesterol/isolated APOA1. HDL particles were further purified by gel filtration chromatography using a Sephacryl S300 column (GE Healthcare). 


\section{Synthesis and use of photocholesterol to label PON1 residues}

Photocholesterol was synthesized by conversion of 6 -keto- $5 \alpha$-cholestan$3 \beta$-ol (Sigma-Aldrich) to 6 -azi- $5 \alpha$-cholestan-3 $\beta$-ol (65), and purity was established by both NMR and mass spectrometric analyses. nHDL containing the photocholesterol probe was prepared as above, but included photocholesterol instead of cholesterol. Photocholesterol containing nHDL was maintained under a foil wrapper in brown glass vials until use. Photolysis was performed on the photo-HDL-PON1 complexes (molar ratio of 1:1) at room temperature in the dark using a UV lamp at $350 \mathrm{~nm}$. After delipidation of the photo-HDL-PON1 complex, photolabeled proteins were subjected to LC/MS/MS analysis using an LTQ-MS system (Thermo Fisher Scientific) for the identification of photocholesterol-labeled peptides.

\section{Proteomics analyses of PON1}

Sample preparations and LC-ESI-MS/MS. HDL was precipitated by chloroform/methanol (2:1 vol/vol), reduced with DTT, alkylated with iodoacetamide, and digested with sequencing grade trypsin (Promega). Peptides were analyzed on a hybrid LTQ Orbitrap mass spectrometer (Thermo Fisher Scientific) interfaced with a nanoelectrospray ion source. Peptides were separated on a reverse-phase column using an acetonitrile and water solvent system containing $0.2 \%$ formic acid with a flow rate of $200 \mathrm{nl} /$ minute. Up to 5 data-dependent $\mathrm{MS}^{2}$ spectra of the most abundant doubly or triply charged precursor signals were acquired in the linear ion trap using collision-induced dissociation. Peak lists were generated using either Bioworks Browser 3.2 (Thermo Fisher Scientific) or Mascot Distiller software 2.3.2 (Matrix Science). The resulting Unified Search Files (*.srf) were searched against the UniProt FASTA of APOA1 (P02647 [25-267]), while Mascot Generic Files ( ${ }^{*}$.mgf) were searched using the Mascot server v2.2 (1) against a human protein database downloaded from the European Bioinformatic Institute (EBI) (release date: 19/01/2010). Only strictly tryptic peptides (66) with a maximum of 1 missed cleavage site were allowed in the database searches. Monoisotopic precursor ions were searched with a tolerance of $6 \mathrm{ppm}$, with $0.5 \mathrm{Da}$ for the fragment ions in the data obtained from the hybrid LTQ Orbitrap mass spectrometer. The signal tolerance for the ion trap mass spectrometer was 1.0 Da for the precursor ion and $0.5 \mathrm{Da}$ for the fragment ions. Unidentified fragment ions in all fragmentation spectra were manually validated using Protein Prospector (University of California) $(67,68)$. H/D exchange and tandem mass spectrometric studies were performed as described previously $(23,43)$.

Quantification of oxidized paraoxonase peptides. Two PON1 peptides (IFFYDSENPPASEVLR; VVAEGFDFANGINISPDGK) that were not modified and readily observable were selected as reference peptides, and extracted ion chromatograms were obtained from these peptides. Extracted ion chromatograms were also obtained from modified peptides containing methionine oxidation and their corresponding unmodified parent peptides. The chromatographic peak area ratio was calculated as the peak area of oxidized or parent unmodified peptide divided by the peak area of the reference peptide.

Effect of methionine sulfoxide reductase on PON1 activity. HDL was incubated with the $\mathrm{MPO} / \mathrm{H}_{2} \mathrm{O}_{2} / \mathrm{Cl}$ system at a molar ratio of $\mathrm{H}_{2} \mathrm{O}_{2} /$ APOA1 (50:1, $\mathrm{mol} / \mathrm{mol}$ ) at $37^{\circ} \mathrm{C}$ for 1 hour in $10 \mathrm{mM}$ sodium phosphate buffer ( $\mathrm{pH} 7.4$ ) containing $100 \mathrm{M}$ DTPA. For the MPO- $\mathrm{H}_{2} \mathrm{O}_{2} \mathrm{Cl}$ system, the reaction mixture was supplemented with $50 \mathrm{nM} \mathrm{MPO}, 100 \mathrm{mM} \mathrm{NaCl}$, and $250 \mu \mathrm{M}$ $\mathrm{H}_{2} \mathrm{O}_{2}$. Oxidation was terminated by the addition of $5 \mathrm{mM}$ methionine. For the reduction reaction, oxidized $\mathrm{HDL}(5 \mu \mathrm{M}$ APOA 1$)$ was incubated with $1 \mu \mathrm{M}$ methionine sulfoxide reductase (MsrA) and $1 \mu \mathrm{M} \mathrm{MsrB} \mathrm{(both} \mathrm{from}$ Jena Bioscience) for 2 hours at $37^{\circ} \mathrm{C}$ in Tris- $\mathrm{HCl}$ buffer $(25 \mathrm{mM}, \mathrm{pH} 7.4)$ containing $15 \mathrm{mM}$ dithiothreitol. Paraoxonase activity for the MsrA study was measured by UV spectrophotometry in a 96-well plate format (Nunclon Surface) using paraoxon (Sigma-Aldrich) as a substrate. HDL (100 $\mu \mathrm{g}$ protein per well) was diluted in a reaction mixture containing $10 \mathrm{mM}$ Tris$\mathrm{HCl}$ ( $\mathrm{pH} 8.0$ ), $1 \mathrm{M}$ sodium chloride and $2 \mathrm{mM}$ calcium chloride. The reaction was initiated at $24^{\circ} \mathrm{C}$ by the addition of $1.5 \mathrm{mM}$ paraoxon. Data are the mean $\pm \mathrm{SD}$ of triplicate determinations.

Immunoprecipitation of HDL (APOA1), MPO, and PON1 in human plasma. Human IgG was depleted from human plasma by a protein A column (Pierce) initially to decrease nonspecific binding proteins. Human IgGdepleted plasma $(250 \mu \mathrm{l})$ spiked with isolated human MPO $(60 \mu \mathrm{g})$ and isolated human PON1 (60 $\mu \mathrm{g})$ was precleared again using Pansorbin cells. Precleared plasma (40 $\mu \mathrm{l})$ was used for immunoprecipitation studies with the indicated IgGs (mAb anti-human APOA1; G45615M; BIODESIGN International); mouse mAb against human MPO (ab51065; Abcam), or no antibody as a control. For endogenous PON1 immunoprecipitation, 2 milliliters of human IgG-depleted plasma spiked with $100 \mu \mathrm{g}$ MPO was precleared with Pansorbin cells. Rabbit mAb anti-PON1 (2835-1; Epitomics) was used to immunoprecipitate PON1.

Surface plasmon resonance. Measurements of apparent dissociation constants between nHDL, MPO, or PON1 were performed using a BIAcore 2000 SPR Biosensor (Biacore) following the methods of Jin et al. (69) with modifications. Briefly, 8,000 resonance units of anti-APOA1 antibody (Biodesign) or 2,000 resonance units of MPO were immobilized on a CM5 sensor chip. nHDL surfaces were generated by injection of $7 \mu \mathrm{M}$ nHDL over the anti-APOA1 antibody surface. The apparent dissociation constants were obtained by fitting background-subtracted SPR binding data to the 1:1 binding with a drifting baseline model using BIAevaluation software, (version 4.0; Biacore).

\section{Statistics}

Statistical significance of differences was determined by a 2-tailed Student's $t$-test or ANOVA where appropriate. Spearman's rank correlation coefficients were calculated to test the correlation between PON1 activity and protein-bound 3-ClTyr levels. $P$ values less than 0.05 were considered statistically significant.

\section{Study approval}

All participants gave written informed consent, and the IRB of the Cleveland Clinic Foundation approved the study protocol. Clinical investigations were conducted in accordance with the Declaration of Helsinki principles. All animal studies were performed using approved protocols from the Animal Research Committee of the Cleveland Clinic.

\section{Acknowledgments}

This study was supported by NIH grants P01HL098055, P01HL076491, R01 HL094322-01A1, R01 HL17964, and 2P01 HL030568-26A1. This work was also supported in part by a grant from the LeDucq Foundation and the Swiss National Research Foundation (138486). S.L. Hazen is also partially supported by a gift from the Leonard Krieger fund. Mass spectrometric studies were performed on instruments housed in a facility in part supported through an AB SCIEX Center of Innovation award.

Received for publication October 22, 2012, and accepted in revised form May 23, 2013.

Address correspondence to: Stanley L. Hazen, Cleveland Clinic, Mail Code NC10, 9500 Euclid Avenue, Cleveland, Ohio 44195, USA. Phone: 216.444.9353; Fax: 216.444.9404; E-mail: hazens@ccf.org.

Zhiping Wu's present address is: St. Jude Children's Research Hospital, Memphis, Tennessee, USA. 
1. Rye KA, Bursill CA, Lambert G, Tabet F, Barter PJ. The metabolism and anti-atherogenic properties of HDL. J Lipid Res. 2009;50(suppl):S195-S200.

2. Degoma EM, Rader DJ. Novel HDL-directed pharmacotherapeutic strategies. Nat Rev Cardiol. 2011;8(5):266-277.

3. Sattler W, Christison J, Stocker R. Cholesterylester hydroperoxide reducing activity associated with isolated high- and low-density lipoproteins. Free Radic Biol Med. 1995;18(3):421-429.

4. VanLenten BJ, et al. Anti-inflammatory HDL becomes pro-inflammatory during the acute phase response - loss of protective effect of HDL against LDL oxidation in aortic wall cell cocultures. J Clin Invest. 1995;96(6):2758-2767.

5. Mineo C, Yuhanna IS, Quon MJ, Shaul PW. High density lipoprotein-induced endothelial nitricoxide synthase activation is mediated by Akt and MAP kinases. J Biol Chem. 2003;278(11):9142-9149.

6. Undurti A, Huang Y, Lupica JA, Smith JD, DiDonato JA, Hazen SL. Modification of high density lipoprotein by myeloperoxidase generates a pro-inflammatory particle. J Biol Chem. 2009; 284(45):30825-30835.

7. Besler C, et al. Mechanisms underlying adverse effects of HDL on eNOS-activating pathways in patients with coronary artery disease. J Clin Invest. 2011;121(7):2693-2708.

8. Garner B, Waldeck AR, Witting PK, Rye KA, Stocker P. Oxidation of high density lipoproteins - II. Evidence for direct reduction of lipid hydroperoxides by methionine residues of apolipoproteins $\mathrm{AI}$ and AII. J Biol Chem. 1998;273(11):6088-6095.

9. Karlsson H, Leanderson P, Tagesson C, Lindahl M. Lipoproteomics II: Mapping of proteins in high-density lipoprotein using two-dimensional gel electrophoresis and mass spectrometry. Proteomics. 2005;5(5):1431-1445.

10. Rezaee F, Casetta B, Levels JHM, Speijer D, Meijers JCM. Proteomic analysis of high-density lipoprotein. Proteomics. 2006;6(2):721-730.

11. Alwaili $\mathrm{K}$, et al. The HDL proteome in acute coronary syndromes shifts to an inflammatory profile. Biochim Biophys Acta. 2012;1821(3):405-415.

12. Davidson W, Silva R, Chantepie S, Lagor W, Chapman M, Kontush A. Proteomic analysis of defined HDL subpopulations reveals particle-specific protein clusters: relevance to antioxidative function. Arterioscler Thromb Vasc Biol. 2009;29(6):870-876.

13. Vaisar T, et al. Shotgun proteomics implicates protease inhibition and complement activation in the antiinflammatory properties of HDL. J Clin Invest. 2007;117(3):746-756.

14. Shih DM, et al. Genetic-dietary regulation of serum paraoxonase expression and its role in atherogenesis in a mouse model. J Clin Invest. 1996;97(7):1630-1639.

15. Bhattacharyya T, et al. Relationship of paraoxonase 1 (PON1) gene polymorphisms and functional activity with systemic oxidative stress and cardiovascular risk. JAMA. 2008;299(11):1265-1276.

16. Mackness MI, Arrol S, Durrington PN. Paraoxonase prevents accumulation of lipoperoxides in low-density lipoprotein. FEBS Lett. 1991;286(1-2):152-154.

17. Watson AD, et al. Protective effect of high density lipoprotein associated paraoxonase - inhibition of the biological activity of minimally oxidized low density lipoprotein. J Clin Invest. 1995;96(6):2882-2891.

18. Aviram M, Rosenblat M, Bisgaier CL, Newton RS, Primo-Parmo SL, La Du BN. Paraoxonase inhibits high-density lipoprotein oxidation and preserves its functions - A possible peroxidative role for paraoxonase. J Clin Invest. 1998;101(8):1581-1590.

19. Shih DM, et al. Mice lacking serum paraoxonase are susceptible to organophosphate toxicity and atherosclerosis. Nature. 1998;394(6690):284-287.

20. Tward A, et al. Decreased atherosclerotic lesion formation in human serum paraoxonase transgenic mice. Circulation. 2002;106(4):484-490.
21. Tang WHW, et al. Diminished antioxidant activity of high-density lipoprotein-associated proteins in systolic heart failure. Circ Heart Fail. 2011;4(1):59-64.

22. Tang WHW, et al. Clinical and genetic association of serum paraoxonase and arylesterase activities with cardiovascular risk. Arterioscler Thromb Vasc Biol. 2012;32(11):516-522.

23. Zheng LM, et al. Apolipoprotein A-I is a selective target for myeloperoxidase-catalyzed oxidation and functional impairment in subjects with cardiovascular disease. J Clin Invest. 2004;114(4):529-541.

24. Wang $Z$, et al. Protein carbamylation links inflammation, smoking, uremia and atherogenesis. Nat Med. 2007;13(10):1176-1184.

25 . Rudolph TK, et al. Myeloperoxidase deficiency preserves vasomotor function in humans. Eur Heart $J$. 2012;33(13):1625-1634

26. Abu-Soud HM, Hazen SL. Nitric oxide is a physiological substrate for mammalian peroxidases. J Biol Chem. 2000;275(48):37524-37532.

27. McMillen TS, Heinecke JW, LeBoeuf RC. Expression of human myeloperoxidase by macrophages promotes atherosclerosis in mice. Circulation. 2005;111(21):2798-2804.

28. Brennan ML, et al. Prognostic value of myeloperoxidase in patients with chest pain. $N$ Engl J Med. 2003;349(17):1595-1604.

29. Hazen SL, Heinecke JW. 3-chlorotyrosine, a specific marker of myeloperoxidase-catalyzed oxidation, is markedly elevated in low density lipoprotein isolated from human atherosclerotic intima. J Clin Invest. 1997;99(9):2075-2081.

30. Sugiyama S, Okada Y, Sukhova GK, Virmani R, Heinecke JW, Libby P. Macrophage myeloperoxidase regulation by granulocyte macrophage colony-stimulating factor in human atherosclerosis and implications in acute coronary syndromes. Am J Pathol. 2001;158(3):879-891.

31. Brennan ML, et al. A tale of two controversies defining both the role of peroxidases in nitrotyrosine formation in vivo using eosinophil peroxidase and myeloperoxidase-deficient mice, and the nature of peroxidase-generated reactive nitrogen species. J Biol Chem. 2002;277(20):17415-17427.

32. Sugiyama S, Kugiyama K, Aikawa M, Nakamura S, Ogawa H, Libby P. Hypochlorous acid, a macrophage product, induces endothelial apoptosis and tissue factor expression - involvement of myeloperoxidase-mediated oxidant in plaque erosion and thrombogenesis. Arterioscler Thromb Vasc Biol. 2004;24(7):1309-1314.

33. Zhang RL, Shen ZZ, NauseefWM, Hazen SL. Defects in leukocyte-mediated initiation of lipid peroxidation in plasma as studied in myeloperoxidase-deficient subjects: systematic identification of multiple endogenous diffusible substrates for myeloperoxidase in plasma. Blood. 2002;99(5):1802-1810.

34. Zhang RL, et al. Myeloperoxidase functions as a major enzymatic catalyst for initiation of lipid peroxidation at sites of inflammation. J Biol Chem. 2002;277(48):46116-46122.

35. Zheng L, et al. Localization of nitration and chlorination sites on apolipoprotein A-I catalyzed by myeloperoxidase in human atheroma and associated oxidative impairment in ABCA1-dependent cholesterol efflux from macrophages. J Biol Chem. 2005;280(1):38-47

36. Graner M, James RW, Kahri J, Nieminen MS, Syvanne M, Taskinen MR. Association of paraoxonase- 1 activity and concentration with angiographic severity and extent of coronary artery disease. J Am Coll Cardiol. 2006;47(12):2429-2435.

37. Wu ZP, et al. Double superhelix model of high density lipoprotein. J Biol Chem. 2009;284(52):36605-36619.

38. Gogonea V, et al. The low resolution structure of nascent high density lipoprotein reconstituted with DMPC with and without cholesterol reveals a mechanism for particle expansion. J Lipid Res.
2013;54(4):966-983

39. Gogonea V, et al. Congruency between biophysical data from multiple platforms and molecular dynamics simulation of the double-super helix model of nascent high-density lipoprotein. Biochemistry. 2010;49(34):7323-7343.

40. Gu F, et al. Structures of discoidal high density lipoproteins: a combined computational-experimental approach. J Biol Chem. 2010;285(7):4652-4665.

41. Li L, Li S, Jones MK, Segrest JP. Rotational and hinge dynamics of discoidal high density lipoproteins probed by interchain disulfide bond formation. Biochim Biophys Acta. 2012;1821(3):481-489.

42. Segrest JP, et al. A detailed molecular belt model for apolipoprotein A-I in discoidal high density lipoprotein. J Biol Chem. 1999;274(45):31755-31758.

43. Wu Z, et al. The refined structure of nascent HDL reveals a key functional domain for particle maturation and dysfunction. Nat Struct Mol Biol. 2007;14(9):861-868

44. Wu ZP, et al. The low resolution structure of ApoA1 in spherical high density lipoprotein revealed by small angle neutron scattering. J Biol Chem. 2011;286(14):12495-12508.

45. Silva RA, et al. Structure of apolipoprotein A-I in spherical high density lipoproteins of different sizes. Proc Natl Acad Sci U S A. 2008;105(34):12176-12181.

46. Church RFR, Weiss MJ. Diazirines. II. Synthesis and properties of small functionalized diazirine molecules. Observations on the reaction of a diaziridine with the iodine-iodide ion system. J Org Chem. 1970;35:2465-2471.

47. Harel M, et al. Structure and evolution of the serum paraoxonase family of detoxifying and anti-atherosclerotic enzymes. Nat Struct Mol Biol. 2004; 11(5):412-419.

48. Ben-David M, et al. Catalytic versatility and backups in enzyme active sites: the case of serum paraoxonase 1. J Mol Biol. 2012;418(3-4):181-196.

49. Peng DQ, et al. Apolipoprotein A-I tryptophan substitution leads to resistance to myeloperoxidasemediated loss of function. Arterioscler Thromb Vasc Biol. 2008;28(11):2063-2070.

50. Annema W, et al. Myeloperoxidase and serum amyloid A contribute to impaired in vivo reverse cholesterol transport during the acute phase response but not group IIA secretory phospholipase A(2). J Lipid Res. 2010;51(4):743-754.

51. Shao B, Tang C, Heinecke JW, Oram JF. Oxidation of apolipoprotein A-I by myeloperoxidase impairs the initial interactions with ABCA1 required for signaling and cholesterol export. J Lipid Res. 2010; 51(7):1849-1858

52. Fiedler TJ, Davey CA, Fenna RE. X-ray crystal structure and characterization of halide-binding sites of human myeloperoxidase at 1.8 A resolution. J Biol Chem. 2000;275(16):11964-11971.

53. Shao B, et al. Tyrosine 192 in apolipoprotein A-I is the major site of nitration and chlorination by myeloperoxidase, but only chlorination markedly impairs ABCA1-dependent cholesterol transport. J Biol Chem. 2005;280(7):5983-5993.

54. Aviram $\mathrm{M}$, et al. Human serum paraoxonase (PON 1) is inactivated by oxidized low density lipoprotein and preserved by antioxidants. Free Radic Biol Med. 1999;26(7-8):892-904.

55. Mackness B, Mackness MI, Arrol S, Turkie W, Durrington PN. Effect of the molecular polymorphisms of human paraoxonase (PON1) on the rate of hydrolysis of paraoxon. Br J Pharmacol. 1997; 122(2):265-268

56. Rosenblat M, et al. The catalytic histidine dyad of high density lipoprotein-associated serum paraoxonase-1 (PON1) is essential for PON1-mediated inhibition of low density lipoprotein oxidation and stimulation of macrophage cholesterol efflux. J Biol Chem. 2006;281(11):7657-7665.

57. Rosenblat M, Volkova N, Aviram M. Injection of 
paraoxonase 1 (PON1) to mice stimulates their HDL and macrophage antiatherogenicity. Biofactors. 2011;37(6):462-467.

58. Berrougui H, Loued S, Khalil A. Purified human paraoxonase- 1 interacts with plasma membrane lipid rafts and mediates cholesterol efflux from macrophages. Free Radic Biol Med. 2012;52(8):1372-1381.

59. Charles-Schoeman C, et al. Cholesterol efflux by high density lipoproteins is impaired in patients with active rheumatoid arthritis. Ann Rheum Dis. 2012;71(7):1157-1162.

60. Shiflett AM, Bishop JR, Pahwa A, Hajduk SL. Human high density lipoproteins are platforms for the assembly of multi-component innate immune complexes. J Biol Chem. 2005;280(38):32578-32585.

61. Markwell MA, Haas SM, Bieber LL, Tolbert NE. A modification of the Lowry procedure to simplify protein determination in membrane and lipoprotein samples. Anal Biochem. 1978;87(1):206-210.

62 . Hatch FT. Practical methods for plasma lipoprotein analysis. Adv Lipid Res. 1968;6:1-68.

63. Aharoni A, Gaidukov L, Yagur S, Toker L, Silman I, Tawfik DS. Directed evolution of mammalian paraoxonases PON1 and PON3 for bacterial expression and catalytic specialization. Proc Natl Acad Sci U S A. 2004;101(2):482-487.

64. Matz CE, Jonas A. Micellar complexes of human apolipoprotein A-I with phosphatidylcholines and cholesterol prepared from cholate-lipid dispersions. J Biol Chem. 1982;257(8):4535-4540.

65. Thiele C, Hannah MJ, Fahrenholz F, Huttner WB. Cholesterol binds to synaptophysin and is required for biogenesis of synaptic vesicles. Nat Cell Biol. 2000;2(1):42-49.
66. Olsen JV, Ong S-E, Mann M. Trypsin cleaves exclusively $\mathrm{C}$-terminal to arginine and lysine residues. Mol Cell Proteomics. 2004;3(6):608-614.

67. Peng J, Elias JE, Thoreen CC, Licklider LJ, Gygi SP. Evaluation of multidimensional chromatography coupled with tandem mass spectrometry (LC/ LC-MS/MS) for large-scale protein analysis: the yeast proteome. J Proteome Res. 2003;2(1):43-50.

68. Baumgartel K, Tweedie-Cullen RY, Grossmann J, Gehrig P, Livingstone-Zatchej M, Mansuy IM. Changes in the proteome after Neuronal Zif268 overexpression.J Proteome Res. 2009:8(7):3298-3316.

69. Jin LH, Shieh JJ, Grabbe E, Adimoolam S, Durbin D, Jonas A. Surface plasmon resonance biosensor studies of human wild-type and mutant lecithin cholesterol acyltransferase interactions with lipoproteins. Biochemistry. 1999;38(47):15659-15665. 